TRANSACTIONS OF THE

AMERICAN MATHEMATICAL SOCIETY

Volume 349, Number 8, August 1997, Pages 3277-3302

S 0002-9947(97)01809-6

\title{
ON THE SECOND ADJUNCTION MAPPING. THE CASE OF A 1-DIMENSIONAL IMAGE
}

\author{
MAURO C. BELTRAMETTI AND ANDREW J. SOMMESE
}

\begin{abstract}
Let $\widehat{L}$ be a very ample line bundle on an $n$-dimensional projective manifold $\widehat{X}$, i.e., assume that $\widehat{L} \approx i^{*} \mathcal{O}_{\mathbb{P}^{N}}(1)$ for some embedding $i: \widehat{X} \hookrightarrow \mathbb{P}^{N}$. In this article, a study is made of the meromorphic map, $\widehat{\varphi}: \widehat{X} \rightarrow \Sigma$, associated to $\left|K_{\widehat{X}}+(n-2) \widehat{L}\right|$ in the case when the Kodaira dimension of $K_{\widehat{X}}+(n-2) \widehat{L}$ is $\geq 3$, and $\hat{\varphi}$ has a 1-dimensional image. Assume for simplicity that $n=$ 3 . The first main result of the paper shows that $\widehat{\varphi}$ is a morphism if either $h^{0}\left(K_{\widehat{X}}+\widehat{L}\right) \geq 7$ or $\kappa(\widehat{X}) \geq 0$. The second main result of this paper shows that if $\kappa(\widehat{X}) \geq 0$, then the genus, $g(f)$, of a fiber, $f$, of the map induced by $\widehat{\varphi}$ on hyperplane sections is $\leq 6$. Moreover, if $h^{0}\left(K_{\widehat{X}}+\widehat{L}\right) \geq 21$ then $g(f) \leq 5$, a connected component $F$ of a general fiber of $\hat{\varphi}$ is either a $K 3$ surface or the blowing up at one point of a $K 3$ surface, and $h^{1}\left(\mathcal{O}_{\widehat{X}}\right) \leq 1$. Finally the structure of the finite to one part of the Remmert-Stein factorization of $\widehat{\varphi}$ is worked out.
\end{abstract}

\section{INTRODUCTION}

Let $\widehat{L}$ be a very ample line bundle on an $n$-dimensional projective manifold $\widehat{X}$, i.e., assume that $\widehat{L} \approx i^{*} \mathcal{O}_{\mathbb{P}^{N}}(1)$ for some embedding $i: \widehat{X} \hookrightarrow \mathbb{P}^{N}$. In this article we study the structure of the meromorphic map, $\widehat{\varphi}: \widehat{X} \rightarrow \Sigma$, associated to $\left|K_{\widehat{X}}+(n-2) \widehat{L}\right|$ in the case when the Kodaira dimension of $K_{\widehat{X}}+(n-2) \widehat{L}$ is $\geq 3$, and $\widehat{\varphi}$ has a 1-dimensional image. For simplicity we assume in most of this introduction that $n=3$. The case when $n \geq 4$ easily reduces to this case and is discussed at the end of this article. For some results in the same direction when $\widehat{X}$ is a surface of general type, we refer the reader to Beauville's article [2], which was one of the motivations for this article.

In [17] it is shown that except for a short and well understood list of degenerate examples, the pairs, $(\widehat{X}, \widehat{L})$, with $\widehat{L}$ a very ample line bundle on a 3 -dimensional projective manifold, have the Kodaira dimension of $K_{\widehat{X}}+\widehat{L}$ equal to 3 , i.e., there exists some positive integer $t$ such that the map associated to $\left|t\left(K_{\widehat{X}}+\widehat{L}\right)\right|$ is birational. It is moreover shown that except for this short list there is the "first reduction" morphism $\pi: \widehat{X} \rightarrow X$ expressing $\widehat{X}$ as the blowup of a projective manifold $X$ at a finite set $B$, and such that:

a) $K_{\widehat{X}}+2 \widehat{L} \cong \pi^{*}\left(K_{X}+2 L\right)$, where $L:=\left(\pi_{*} \widehat{L}\right)^{* *}$ is an ample line bundle, and $K_{X}+2 L$ is ample;

Received by the editors January 11, 1996.

1991 Mathematics Subject Classification. Primary 14E35, 14C20, 14J40.

(C)1997 American Mathematical Society 
b) $K_{X}+L$ is nef and big, i.e., $\left(K_{X}+L\right) \cdot C \geq 0$ for every effective curve $C \subset X$ and $\left(K_{X}+L\right)^{3}>0$.

The hope that except for a few examples, $K_{X}+L$ is not just nef, but spanned at all points by global sections is supported by a number of results (see, e.g., the Introduction of [5]). There is a well known counterexample [12] of a Del Pezzo threefold with $L$ very ample, $L^{3}=27$, and with $K_{X}+L$ ample but not everywhere spanned. This is in line with a conjecture of the second author [18] that $K_{X}+L$ is spanned if the Kodaira dimension of $X$ is nonnegative. This is in fact one of the main open problems in adjunction theory.

Trying to better understand the behavior of the adjoint bundle $K_{X}+L$ led us to the following strong result proved in $[5,(1.2),(3.3),(3.4)]$.

Theorem. Let $\widehat{L}$ be a very ample line bundle on a projective manifold $\widehat{X}$ of dimension 3. If the Kodaira dimension of $K_{\widehat{X}}+\widehat{L}$ is 3 , then $h^{0}\left(K_{\widehat{X}}+\widehat{L}\right) \geq 2$. If the Kodaira dimension of $\widehat{X}$ is nonnegative, then $h^{0}\left(K_{\widehat{X}}+\widehat{L}\right) \geq 5$ with equality only if $(\widehat{X}, \widehat{L})$ is a degree 5 hypersurface of $\mathbb{P}^{4}$.

This paper, which grew out of the above result, is a natural sequel of [5].

Let $\varphi: X \rightarrow \Sigma$ be the rational map associated to the complete linear system $\left|K_{X}+L\right|$. Note that $\operatorname{dim} \Sigma>0$ by the above theorem. In this paper we deal with the case when $\Sigma$ is 1-dimensional.

In $\S \S 1$ and 2 we state some background material and some preliminary results we need.

In $\S \S 3$ and 4 we prove the first main result of the paper, Theorem (4.5), which states that $\varphi$ is a morphism if either $h^{0}\left(K_{X}+L\right) \geq 7$ or $\kappa(\widehat{X}) \geq 0$. Note also the general fact that $\varphi$ is always a morphism if the normalization of $\Sigma$ is a positive genus curve.

In $\S \S 5$ and 6 we study the structure of the morphism $\varphi$. Let $f=F \cap S$ be the transversal intersection of a general fiber $F$ of the connected part of $\varphi$ with a general smooth surface $S$ in $|L|$. In $\S 5$ we show that $g(f) \leq 10$. Further we show that under more restrictive assumptions on $h^{0}\left(K_{X}+L\right)$ we get sharper upper bounds, e.g., if $h^{0}\left(K_{X}+L\right) \geq 10$ then $g(f) \leq 6$ (see Theorem (5.2)).

In $\S 6$ we consider the case when $\kappa(\widehat{X}) \geq 0$. Then by the above $\varphi$ is always a morphism in this case. In the second main result of this paper, Theorem (6.1), we show that $g(f) \leq 6$. Moreover if $h^{0}\left(K_{X}+L\right) \geq 21$, we prove that $g(f) \leq 5, F$ is either a $K 3$ surface or the blowing up at one point of a $K 3$ surface, and $h^{1}\left(\mathcal{O}_{X}\right) \leq 1$. Thus for $h^{0}\left(K_{\widehat{X}}+\widehat{L}\right) \geq 21$ and $\kappa(\widehat{X}) \geq 2$ the map associated to $\left|K_{\widehat{X}}+\widehat{L}\right|$ has an image of dimension at least 2 .

In $\S 7$ we find degree bounds for the finite-to-one part $s: C \rightarrow \mathbb{P}_{\mathbb{C}}$ of the RemmertStein factorization, $\varphi=r \circ s$, of $\varphi$ (see (2.1)). It is clear that if $C$ is $\mathbb{P}^{1}$ then $s$ is an embedding, and hence \#s=1. If $C$ has positive arithmetic genus we show in Theorem (7.4) that either $\# s=1$ or $\# s=2$, and if $\# s=2$ then $h^{0}\left(K_{X}+L\right)=2$, $g(f) \leq 4$, and $\chi\left(\mathcal{O}_{S}\right) \leq 2$. In particular, $\# s=1$ if $\kappa(\widehat{X}) \geq 0$ (cf. $(7.5)$ ).

In $\S 8$ we discuss the extension of the results of the paper to the case when $\operatorname{dim} \widehat{X} \geq 4$.

The cases when $\operatorname{dim} \varphi(X)=2,3$ will be treated in future papers.

The methods of the proofs mainly rely on the adjunction theory of 3-folds, the double point formula inequality for 3 -folds in projective space, Tsuji's inequality, and Lefschetz theory. We also use over and over the Hodge index inequalities, 
Castelnuovo's bounds for the genus of a curve in projective space, and the fact that $S$ is a minimal surface of general type.

Both authors would like to thank the Mathematical Institute of the University of Göttingen for support during the summer of 1992, where this project was conceived, and the Mathematics Department of the University of Notre Dame for support in October 1994, when the final research on this paper was carried out.

The second author also thanks the National Science Foundation (NSF Grants DMS 89-21702 and DMS 93-02121) for support.

\section{BACKGROUND MATERIAL}

We refer to our book [6] for all the results on adjunction theory we need in this paper and also for a complete list of references to the original sources.

We work over the complex numbers $\mathbb{C}$. Throughout the paper we deal with smooth, projective varieties $V$. We denote by $\mathcal{O}_{V}$ the structure sheaf of $V$ and by $K_{V}$ the canonical bundle. For any coherent sheaf $\mathcal{F}$ on $V, h^{i}(\mathcal{F})$ denotes the complex dimension of $H^{i}(V, \mathcal{F})$.

Let $L$ be a line bundle on $V$. The line bundle $L$ is said to be numerically effective (nef, for short) if $L \cdot C \geq 0$ for all effective curves $C$ on $V$. $L$ is said to be big if $\kappa(L)=\operatorname{dim} V$, where $\kappa(L)$ denotes the Kodaira dimension of $L$. If $L$ is nef then this is equivalent to $c_{1}(L)^{n}>0$, where $c_{1}(L)$ is the first Chern class of $L$ and $n=\operatorname{dim} V$.

1.1. Notation. The notation used in this paper is standard from algebraic geometry. Let us only fix the following.

$\approx$ (respectively $\sim$ ), linear (respectively numerical) equivalence of line bundles; $\chi(L)=\sum_{i}(-1)^{i} h^{i}(L)$, the Euler characteristic of a line bundle $L$;

$|L|$, the complete linear system associated with a line bundle $L$ on a variety $V$,

$\Gamma(L)=H^{0}(L)$, the space of the global sections of $L$. We say that $L$ is spanned if it is spanned at all points of $V$ by $\Gamma(L)$;

$e(V)=c_{n}(V)$, the topological Euler characteristic of $V$, for $V$ smooth, where $c_{n}(V)$ is the $n$-th Chern class of the tangent bundle of $V$. If $V$ is a surface, then $e(V)=12 \chi\left(\mathcal{O}_{V}\right)-K_{V} \cdot K_{V}$;

$\kappa(V):=\kappa\left(K_{V}\right)$, the Kodaira dimension, for $V$ smooth;

$\lceil x\rceil$, the smallest integer bigger or equal to a rational number $x$.

Line bundles and divisors are used with little (or no) distinction. Hence we freely switch between the multiplicative and the additive notation.

1.2. Genus formula. For a line bundle $L$ on a variety $V$ of dimension $n$ the sectional genus, $g(L)=g(V, L)$, of $(V, L)$ is defined by

$$
2 g(L)-2=\left(K_{V}+(n-1) L\right) \cdot L^{n-1} .
$$

Note that if $|L|$ contains $n-1$ elements $H_{1}, \ldots, H_{n-1}$ meeting in a reduced irreducible curve $C$, then $g(L)=g(C)=1-\chi\left(\mathcal{O}_{C}\right)$, the arithmetic genus of $C$.

1.3. Reduction. (See, e.g., $[6$, Chapters 7,12$]$.) Let $(\widehat{X}, \widehat{L})$ be a smooth projective variety of dimension $n \geq 2$ polarized with a very ample line bundle $\widehat{L}$. A smooth 
polarized variety $(X, L)$ is called a (first) reduction of $(\widehat{X}, \widehat{L})$ if there is a morphism $\pi: \widehat{X} \rightarrow X$ expressing $\widehat{X}$ as the blowing up of $X$ at a finite set of points, $B$, such that $L:=\left(\pi_{*} \widehat{L}\right)^{* *}$ is ample and $\widehat{L} \approx \pi^{*} L-\left[\pi^{-1}(B)\right]$ or, equivalently, $K_{\widehat{X}}+(n-1) \widehat{L} \approx$ $\pi^{*}\left(K_{X}+(n-1) L\right)$.

Note that there is a one to one correspondence between smooth divisors of $|L|$ which contain the set $B$ and smooth divisors of $|\widehat{L}|$.

Except for an explicit list of well understood pairs $(\widehat{X}, \widehat{L})$ (see in particular $[6$, $\S \S 7.2,7.3,7.4,7.5])$ we can assume:

a) $K_{\widehat{X}}+(n-1) \widehat{L}$ is spanned and big, and $K_{X}+(n-1) L$ is very ample. Note that in this case this reduction, $(X, L)$, is unique up to isomorphism. We will refer to it as the first reduction of $(\widehat{X}, \widehat{L})$.

b) $K_{X}+(n-2) L$ is nef and big, for $n \geq 3$.

Note that $h^{0}\left(K_{\widehat{X}}+(n-2) \widehat{L}\right)=h^{0}\left(K_{X}+(n-2) L\right)$. Indeed one has (see [6, (7.6.1)])

$$
\Gamma\left(a K_{\widehat{X}}+b \widehat{L}\right) \cong \Gamma\left(a K_{X}+b L\right)
$$

for integers $a, b$ with $b \leq a(n-1)$.

Note also that $\kappa\left(K_{\widehat{X}}+(n-2) \widehat{L}\right) \geq 0$ implies $\kappa\left(K_{\widehat{X}}+(n-1) \widehat{L}\right)=n$, so that the first reduction $(X, L)$ exists and $K_{X}+(n-2) L$ is nef (see [6, (7.6.9)]).

1.4. Pluridegrees. Let $(\widehat{X}, \widehat{L}),(X, L)$ be as in (1.3) with $n=3$. Define the pluridegrees, for $j=0,1,2,3$, by

$$
\widehat{d}_{j}:=\left(K_{\widehat{X}}+\widehat{L}\right)^{j} \cdot \widehat{L}^{3-j} \text { and } d_{j}:=\left(K_{X}+L\right)^{j} \cdot L^{3-j} .
$$

If $\gamma$ denotes the number of points blown up under $\pi: \widehat{X} \rightarrow X$, then because $K_{\widehat{X}}+\widehat{L} \cong \pi^{*}\left(K_{X}+L\right)+\sum_{i} E_{i}$, the invariants $\widehat{d}_{j}, d_{j}$ are related by

$$
\widehat{d}_{0}=d_{0}-\gamma ; \widehat{d}_{1}=d_{1}+\gamma ; \widehat{d}_{2}=d_{2}-\gamma ; \widehat{d}_{3}=d_{3}+\gamma .
$$

We put $\widehat{d}:=\widehat{d}_{0}, d:=d_{0}$. If $K_{X}+L$ is nef, by the generalized Hodge index theorem (see, e.g., $[6,(2.5 .1),(13.1)],[7,(1.2)])$ one has

$$
d_{1}^{2} \geq d d_{2} \text { and } d_{2}^{2} \geq d_{1} d_{3},
$$

and the parity lemma (13.1.1) of [6] says that

$$
d \equiv d_{1} \bmod (2) ; d_{2} \equiv d_{3} \bmod (2) .
$$

Moreover if $K_{X}+L$ is nef and big, the numbers $d_{j}$ are positive.

We need the following versions of the double point formula (for the proofs and full details see $[5,(0.5)]$ and also $[6,(8.2),(13.1)])$.

Proposition 1.5. Let $(\widehat{X}, \widehat{L})$ be a smooth projective 3 -fold, polarized with a very ample line bundle, $\widehat{L}$. Assume that $\kappa\left(K_{\widehat{X}}+\widehat{L}\right) \geq 2$. Let $(X, L)$ and $\pi: \widehat{X} \rightarrow X$ be the first reduction and the first reduction map respectively. Let $\widehat{d}_{j}, d_{j}, 0 \leq j \leq 3$, be the pluridegrees of $(\widehat{X}, \widehat{L})$ and $(X, L)$ respectively. Let $\gamma$ be the number of points blown up by $\pi$. Let $\widehat{S}$ be a smooth element in $|\widehat{L}|$. Then

$$
44 h^{0}\left(K_{\widehat{X}}+\widehat{L}\right)+58 \chi\left(\mathcal{O}_{\widehat{S}}\right)+2 h^{0}\left(K_{\widehat{X}}\right)+4 \geq 12 d_{2}+17 d_{1}+d_{3}+(20-\widehat{d}) \widehat{d}+5 \gamma .
$$


Lemma 1.6. Let $(\widehat{X}, \widehat{L})$ be as in (1.3) with $n=3$, and let $\widehat{S}$ be a smooth element of $|\widehat{L}|$. Assume that $\Gamma(\widehat{L})$ embeds $\widehat{X}$ in $\mathbb{P}^{N}$ with $N \geq 5$. Then

$$
\widehat{d}^{2}-5 \widehat{d}-10(g(\widehat{L})-1)+12 \chi\left(\mathcal{O}_{\widehat{S}}\right) \geq 2 K_{\widehat{S}} \cdot K_{\widehat{S}}
$$

with equality if $N=5$.

Corollary 1.7. Let $(\widehat{X}, \widehat{L})$ be a smooth projective 3 -fold, polarized with a very ample line bundle, $\widehat{L}$. Let $(X, L)$ and $\pi: \widehat{X} \rightarrow X$ be the first reduction and the first reduction map respectively. Let $\widehat{d}:=\widehat{L}^{3}$ and let $d_{j}, 0 \leq j \leq 3, d:=d_{0}$, be the pluridegrees of $(X, L)$. Let $\gamma$ be the number of points blown up by $\pi$. Let $S$ be a smooth element in $|L|$, corresponding to a smooth element in $|\widehat{L}|$. Then, if $d+\widehat{d} \geq 7$, we have

$$
d^{2}-5 d-10(g(L)-1)+12 \chi\left(\mathcal{O}_{S}\right) \geq K_{S} \cdot K_{S} .
$$

Proof. From Lemma (1.6) we get

$$
(d-\gamma)(d-5-\gamma)-10(g(L)-1)+12 \chi\left(\mathcal{O}_{S}\right) \geq 2 d_{2}-2 \gamma,
$$

or $d(d-5)-10(g(L)-1)+12 \chi\left(\mathcal{O}_{S}\right)+\gamma(\gamma+7-2 d) \geq 2 d_{2}$. Since $\gamma+7-2 d=$ $7-d-\widehat{d} \leq 0$, we are done.

Q.E.D.

The following inequalities follow from the log version of the usual Yau inequality given in $[19, \S 5]$ (see $[5,(0.6)]$ or $[6,(13.1 .7),(13.1 .8)]$ for full details).

Proposition 1.8 (Tsuji's inequality). Let $(\widehat{X}, \widehat{L})$ be a smooth projective 3-fold, polarized with a very ample line bundle, $\widehat{L}$. Assume that $\kappa\left(K_{\widehat{X}}+\widehat{L}\right) \geq 0$. Let $(X, L)$ be the first reduction of $(\widehat{X}, \widehat{L})$. Let $S$ be a smooth element of $|L|$. Then we have $\left(K_{X}+L\right)^{3}+\frac{8}{3} K_{S} \cdot L_{S} \leq 32\left(2 h^{0}\left(K_{X}+L\right)-\chi\left(\mathcal{O}_{S}\right)\right)$, or

$$
h^{0}\left(K_{X}+L\right) \geq \frac{d_{3}}{64}+\frac{d_{1}}{24}+\frac{\chi\left(\mathcal{O}_{S}\right)}{2} .
$$

1.9. Castelnuovo bound. Let $C$ be a reduced, irreducible projective curve. Assume that $\psi: C \rightarrow \mathbb{P}^{N}$ is a generically one-to-one morphism, and that $\psi(C)$ does not lie in any hyperplane. Let $d$ denote the degree of $\psi(C)$ in $\mathbb{P}^{N}$. Let $g(C)$ be the arithmetic genus of $C$. Then Castelnuovo's lemma (see, e.g., [8, Theorem 3.7]) reads

$$
g(C) \leq \operatorname{Castel}(d, N):=\left[\frac{d-2}{N-1}\right]\left(d-N-\left(\left[\frac{d-2}{N-1}\right]-1\right) \frac{N-1}{2}\right),
$$

where $[x]$ means the greatest integer $\leq x$.

1.10. Threefolds of log-general type. Let $(\widehat{X}, \widehat{L}),(X, L)$ be as in (1.3) with $n=3$ and let $\widehat{d}_{j}, d_{j}, j=0,1,2,3$, be the pluridegrees as in (1.4). We say that $(\widehat{X}, \widehat{L})$ is of log-general type if $K_{X}+L$ is nef and big. Hence in particular the numbers $d_{j}$ are positive in this case.

Let $\widehat{S}$ be a smooth element of $|\widehat{L}|$ and $S$ the corresponding smooth surface in $|L|$. Then by the adjunction formula $K_{S}$ is nef. Furthermore $K_{S}$ is also big, since some multiple of $K_{S}$ is the pullback of some ample divisor under the restriction of $\varphi$ to $S$. Then $S$ is a minimal surface of general type. Hence

$$
d_{2}=K_{S} \cdot K_{S}<9 \chi\left(\mathcal{O}_{S}\right) .
$$


Indeed the Miyaoka inequality yields $d_{2} \leq 9 \chi\left(\mathcal{O}_{S}\right)$. Note that the equality cannot happen. Otherwise $S$ is a ball quotient and hence a $K(\Pi, 1)$, which would contradict $[15,(1.3)]$. We will often use (4) in the form

$$
\frac{\chi\left(\mathcal{O}_{S}\right)}{2} \geq \frac{d_{2}+1}{18} \text {. }
$$

Note that since $\chi\left(\mathcal{O}_{S}\right)$ is an integer we have the stronger integrality relation

$$
\chi\left(\mathcal{O}_{S}\right) \geq\left\lceil\frac{d_{2}+1}{9}\right\rceil .
$$

When $\kappa(X) \geq 0$, we know from [16, (1.5), (3.1)] (see also [6, (13.1.3)]) that $d_{3} \geq$ $d_{2} \geq d_{1} \geq d$. Note that if $\kappa(X) \geq 0$, then $(\widehat{X}, \widehat{L})$ is of log-general type. Indeed, if $h^{0}\left(t K_{X}\right)>0$ for some positive integer $t$, then $t\left(K_{X}+L\right)$ gives a birational embedding on a Zariski open set by sections of $\Gamma(L)$, and thus $\kappa\left(K_{X}+L\right)=3$.

We also need the following general result.

Lemma 1.11. Let $V$ be an irreducible variety of dimension $n \geq 3$. Let $L$ be a line bundle on $V$. Assume that $L$ is very ample outside of a finite set of points and let $\ell$ be a line on $V$, i.e., $\ell \cdot L=1$. Let $D$ be an effective divisor on $V$. If $\ell \cdot D \geq x$, then $L^{n-1} \cdot D \geq \ell \cdot D \geq x$.

Proof. We can write $L^{n-1}=\ell+\mathcal{R}$, where $\mathcal{R}$ moves and hence $\mathcal{R} \cdot D \geq 0$. Therefore $L^{n-1} \cdot D \geq \ell \cdot D \geq x$.

Q.E.D.

For any further background material we refer to [5].

\section{Notation AND GENERAL SET-UP}

Let $(\widehat{X}, \widehat{L})$ be a smooth threefold polarized with a very ample line bundle $\widehat{L}$. Assume that $(\widehat{X}, \widehat{L})$ is of log-general type, which implies that the first reduction $(X, L), \pi: \widehat{X} \rightarrow X$, of $(\widehat{X}, \widehat{L})$ exists and that $K_{X}+L$ is nef and big. Let $\widehat{S}$ be a general element of $|\widehat{L}|$ and let $S$ be the corresponding smooth surface in $|L|$. Recall that the restriction $\pi_{\widehat{S}}: \widehat{S} \rightarrow S$ maps $\widehat{S}$ onto its minimal model, $S$, and $S$ is of general type. Recall that $h^{0}\left(K_{\widehat{X}}+\widehat{L}\right)=h^{0}\left(K_{X}+L\right)$ by (1).

Let $\varphi: X \rightarrow \mathbb{P}^{n}$ be the rational map associated to $\left|K_{X}+L\right|$. Let $\Sigma:=\varphi(X)$. Since from [5, (1.2)] we know that $h^{0}\left(K_{X}+L\right) \geq 2$, we have $\operatorname{dim} \Sigma \geq 1$. Since we deal in this paper with the case when $\Sigma$ is a curve, we can assume that $h^{0}\left(K_{X}\right)=0$. Indeed if $h^{0}\left(K_{X}\right)>0$, a section of $\Gamma\left(K_{X}\right)$ gives an embedding $\Gamma(L) \hookrightarrow \Gamma\left(K_{X}+L\right)$. Since $L$ is very ample off a finite set of points, $\left|K_{X}+L\right|$ defines a birational map.

2.1. General construction. With the notation as above, let $\Gamma \subset X \times \Sigma$ be the graph of $\varphi$. Let $\rho: \bar{X} \rightarrow \Gamma$ be a smooth resolution of $\Gamma$. Let $\sigma: \bar{X} \rightarrow X$ be the composition of $\rho$ with the product projection $X \times \Sigma \rightarrow X$, and let $\bar{\varphi}: \bar{X} \rightarrow \Sigma$ be the composition of $\rho$ with the product projection $X \times \Sigma \rightarrow \Sigma$. Let $\bar{\varphi}=s \circ \bar{r}$ be the Remmert-Stein factorization of $\bar{\varphi}$, where $\bar{r}$ is a morphism with connected fibers. Thus we have a commutative diagram

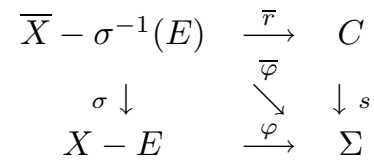


where $E$ is the indeterminacy locus of $\varphi$. Then there exists a rational map $r:=$ $\bar{r} \circ \sigma^{-1}: X \rightarrow C$ such that $\varphi=s \circ r$. We say that $s \circ r$ is the Remmert-Stein factorization of the rational map $\varphi$. Note also that the general fiber, $F$, of $r$ is irreducible. If $S$ is a smooth general member of $|L|$, then $F, S$ meet transversely in an irreducible curve,

$$
f:=F \cap S .
$$

We have the following general fact:

$$
f \cdot f=0 \text { on } S \text { if and only if } \varphi \text { is a morphism. }
$$

To see this, assume that $\varphi$ is not a morphism and let $E \neq \emptyset$ be the indeterminacy locus of $\varphi$. We claim that $E$ does not contain an isolated point. Indeed, assume otherwise and let $x \in E$ be an isolated point. Then there exist two divisorial fibers of $\varphi$ meeting only at $x$, and this is not possible for dimensional reasons. It thus follows that $S$ meets $E$. This shows that if the restriction map $\varphi_{S}: S \rightarrow \Sigma$ is a morphism, then $\varphi: X \rightarrow \Sigma$ is a morphism. Since $f \cdot f=0$ on $S$ implies that $\varphi_{S}$ is a morphism, we are done.

We will use the following general result.

Lemma 2.2. Let $X$ be an $n$-dimensional smooth projective variety and let $\varphi: X \rightarrow$ $C$ be a rational (i.e., meromorphic) map onto a smooth curve of genus $g(C)>0$. Then $\varphi$ is a morphism.

Proof. Let $\bar{\varphi}$ be the morphism associated to $\varphi$ constructed as in (7). Then $\bar{\varphi}=\varphi \circ \sigma$, where $\sigma: \bar{X} \rightarrow X$ is a smooth resolution of the graph $\Gamma \subset X \times C$ of $\varphi$. We have a commutative diagram

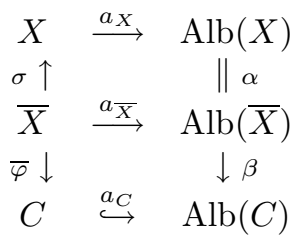

where $\beta$ is induced from the morphism $\bar{\varphi}, \alpha$ is an isomorphism since $\bar{X}, X$ are birational, $a_{\bar{X}}, a_{X}, a_{C}$ are the Albanese mappings, and $a_{C}$ is an embedding since $C$ has positive genus. There exists a Zariski open set $U \subset X$ such that $U$ maps holomorphically onto $C$ under $\bar{\varphi} \circ \sigma^{-1}: U \rightarrow C$. Moreover $\bar{\varphi} \circ \sigma^{-1}: U \rightarrow C$ extends to $X$ via $\beta \circ \alpha \circ a_{X}$. It thus follows that $\left(\beta \circ \alpha \circ a_{X}\right)(X)=C$. Then $\beta \circ \alpha \circ a_{X}=\varphi$ is a morphism.

Q.E.D.

Thus, by using Lemma (2.2), to show that $\varphi: X \rightarrow \Sigma$ is a morphism we can assume that $C \cong \mathbb{P}^{1}$ in (7) and that $\varphi$ Remmert-Stein factors as (cf. diagram (7))

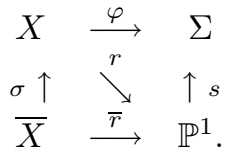

We will denote $\mathcal{K}:=K_{X}+L$. Then $r$ is the meromorphic map given by the complete linear system $|\mathcal{K}|$. It is a general fact, see, e.g., [9, Chap. II, 7.17.3], that there exists a spanned line bundle $\overline{\mathcal{K}}$ on $\bar{X}$ such that

$$
\overline{\mathcal{K}} \approx \sigma^{*} \mathcal{K}-Z
$$


for some effective divisor $Z$ on $\bar{X}$ and such that $\sigma_{*} \overline{\mathcal{K}} \approx \mathcal{K} \otimes \mathcal{J}$, where $\mathcal{J}$ is the ideal sheaf of $\sigma(Z)$ in $X$, and $\Gamma(\mathcal{K}) \cong \Gamma(\overline{\mathcal{K}})(\cong \Gamma(\mathcal{K} \otimes \mathcal{J}))$. Note that $\bar{r}$ is the morphism given by $|\overline{\mathcal{K}}|$ and $\overline{\mathcal{K}} \approx \bar{r}^{*} \mathcal{O}_{\mathbb{P}^{1}}(t)$ for some positive integer $t$. Then $h^{0}(\mathcal{K})=h^{0}(\overline{\mathcal{K}})=$ $t+1$, and

$$
\mathcal{K} \approx t F+\mathcal{E}
$$

where $F$ is the general fiber of $r$ and $\mathcal{E}$ is an effective divisor which coincides with the divisorial component of $\sigma(Z)$. To show (10) note that $\overline{\mathcal{K}} \approx t \bar{F}$, where $\bar{F}$ is a general fiber of $\bar{r}$. Then, by $(9), \sigma^{*} \mathcal{K} \approx t \bar{F}+Z$, which gives (10) by applying $\sigma_{*}$.

The next two sections, $\S \S 2,3$, are devoted to showing that, for $h^{0}\left(K_{X}+L\right) \gg 0$, $\varphi$ is a morphism, i.e., by $\bullet$ ) that $f:=F \cap S, S$ a smooth general element of $|L|$, satisfies the condition $f \cdot f=0$ on $S$.

We conclude this section by stating two general bounds we use in the sequel.

Proposition 2.3. Let $(\widehat{X}, \widehat{L})$ be a smooth threefold polarized by a very ample line bundle $\widehat{L}$. Assume that $(\widehat{X}, \widehat{L})$ is of log-general type. Let $(X, L)$ be the first reduction of $(\widehat{X}, \widehat{L})$. Assume further that $\left|K_{X}+L\right|$ gives a rational map, $\varphi$, having a 1 dimensional image. Let $f=F \cap S$ be the transversal intersection of a general fiber of the connected part of $\varphi$ with a general smooth surface $S \in|L|$ as in (8). We have:

1) $L \cdot f \geq 4$;

2) if $\varphi$ is not a morphism (i.e., $f \cdot f>0$ on $S$ ) and $\kappa(X) \geq 0$, then $L \cdot f \geq 5$.

Proof. Let $L_{S}$ be the restriction of $L$ to $S$. If $L_{S} \cdot f=L \cdot f \leq 2$, then there exists a pencil of rational curves, $f$, on $S$; if $L_{S} \cdot f=L \cdot f=3$, then there exists a pencil of rational or elliptic curves, $f$, on $S$. In both cases we contradict the condition $\kappa(S)=2$, which follows from the nefness and bigness assumption on $K_{X}+L$. Thus $L \cdot f \geq 4$.

To show 2), assume $\kappa(X) \geq 0$ and $f \cdot f>0$ on $S$. Let $L \cdot f=4$. Let $g(f)$ be the arithmetic genus of $f$. Then $g(f) \leq 3$ by the Castelnuovo bound (1.9), and therefore

$$
4 \geq 2 g(f)-2=\left(K_{S}+f\right) \cdot f=\left(K_{X}+L+F\right)_{S} \cdot f=K_{X} \cdot f+f^{2}+4 .
$$

This gives $K_{X} \cdot f \leq-f \cdot f \leq-1$. Since $\kappa(X) \geq 0$ this contradicts the fact that $f$ moves in at least a 2-dimensional family to fill up $X$.

Q.E.D.

The following result is not optimal, but it is more than enough for our purposes.

Proposition 2.4. Let $(\widehat{X}, \widehat{L})$ be a smooth threefold polarized by a very ample line bundle $\widehat{L}$. Assume that $(\widehat{X}, \widehat{L})$ is of log-general type. Let $(X, L)$ be the first reduction of $(\widehat{X}, \widehat{L})$. Assume further that $\left|K_{X}+L\right|$ gives a rational map which is not a morphism. Then $\widehat{d}=\widehat{L}^{3} \geq 9$.

Proof. We can assume that $\Gamma(\widehat{L})$ embeds $\widehat{X}$ in $\mathbb{P}^{N}$ with $N \geq 5$. Then by the classification of threefolds of degree $\widehat{d} \leq 8$ due to Okonek and Ionescu (see, e.g., [10] or [4], §6) we see that for a threefold $(\widehat{X}, \widehat{L})$ of log-general type and of degree $\widehat{d} \leq 8$ one has $\widehat{X} \cong X$, and $K_{\widehat{X}}+\widehat{L} \cong K_{X}+L$ is spanned. $\quad$ Q.E.D. 


\section{The CASE $f \cdot f \geq 2$}

Notation as in $\S 2$. In this section we assume that the rational map $\varphi: X \rightarrow \Sigma$ associated to $\left|K_{X}+L\right|, \Sigma$ an irreducible curve, is not a morphism and that $f$ from (8) satisfies $f \cdot f:=(f \cdot f)_{S} \geq 2$ on $S$. By Lemma (2.2) we can assume without loss of generality that $r: X \rightarrow \mathbb{P}^{1}$, where $\varphi=s \circ r$ is the Remmert-Stein factorization of $\varphi$ in the sense of (2.1).

3.1. General relations for $f \cdot f \geq 2$. Let $d:=d_{0}, d_{1}, d_{2}, d_{3}$ be the pluridegrees of $(X, L)$ as in (1.4). Let $h:=h^{0}(\mathcal{K})$ and let $\mathcal{K}_{S}, L_{S}$ be the restrictions of $\mathcal{K}, L$ to a general smooth surface $S \in|L|$ respectively. Note that $\mathcal{K}_{S} \cong K_{S}, \mathcal{K} \cdot f=K_{S} \cdot f$, $L_{S} \cdot f=L \cdot f$. Let $g(f)$ be the arithmetic genus of $f$. Recall that $\mathcal{K} \approx(h-1) F+\mathcal{E}$, $\mathcal{E}$ an effective divisor, by (10). The following relations hold:

i) $L \cdot f \geq\lceil\sqrt{d(f \cdot f)}\rceil \geq 6$;

ii) $\left[\sqrt{d_{2}(f \cdot f)}\right] \leq \mathcal{K} \cdot f \leq d_{2} /(h-1)$;

iii) $d_{1} \geq L \cdot f(h-1) \geq(h-1)\lceil\sqrt{d(f \cdot f)}\rceil$;

iv) $d_{2} \geq(f \cdot f)(h-1)^{2}$;

v) $d_{3} \geq h-1$

vi) $2 g(f)-2=\mathcal{K} \cdot f+f \cdot f$.

Let us now prove the above relations. We use (10) over and over without explicitly referring to it. Let $F$ be a general fiber of the connected part of the rational map $\varphi$ as in (8).

i) Note that by the Hodge index theorem, we have $d(f \cdot f)=L_{S}^{2}(f \cdot f) \leq\left(L_{S} \cdot f\right)^{2}$, which gives $L \cdot f \geq\lceil\sqrt{d(f \cdot f)}\rceil$. Using Proposition (2.4) and $f \cdot f \geq 2$, we obtain the second inequality (see Note added in proof).

ii) From the Hodge index theorem we have $d_{2}(f \cdot f)=K_{S}^{2}(f \cdot f) \leq\left(K_{S} \cdot f\right)^{2}$, and therefore $\mathcal{K} \cdot f \geq\left[\sqrt{d_{2}(f \cdot f)}\right]$. The other inequality follows from $d_{2}=K_{S}^{2}=$ $K_{S} \cdot\left((h-1) f+\mathcal{E}_{S}\right) \geq(h-1) \mathcal{K} \cdot f$.

iii) We have $d_{1}=L \cdot L \cdot \mathcal{K}=(h-1) L \cdot L \cdot F+L \cdot L \cdot \mathcal{E} \geq L \cdot f(h-1)$. Since $L \cdot L \cdot F=L \cdot f$ and $L \cdot \mathcal{E} \geq 0$, the second inequality of iii) now follows from i).

iv) We have $d_{2}=K_{S} \cdot K_{S}=\mathcal{K}_{S} \cdot \mathcal{K}_{S} \geq(h-1) f \cdot\left((h-1) f+\mathcal{E}_{S}\right) \geq(h-1)^{2}(f \cdot f)$.

v) One has $d_{3}=\mathcal{K}^{3}=\mathcal{K} \cdot \mathcal{K} \cdot((h-1) F+\mathcal{E})$. Then the inequality follows by noting that $\mathcal{K} \cdot \mathcal{K} \cdot F \geq 1$, since $\mathcal{K}$ is nef and big and $F$ moves (see, e.g., $[6,(2.1 .2)]$ ) and $\mathcal{K} \cdot \mathcal{K} \cdot \mathcal{E} \geq 0$

vi) This is simply the genus formula for $f$ in $S$, recalling that $\mathcal{K} \cdot f=K_{S} \cdot f$.

We need first to prove a "coarse" preparatory result, which states that for $h:=$ $h^{0}\left(K_{X}+L\right) \geq 9$ the case $f \cdot f \geq 2$ does not occur.

Proposition 3.2 (Coarse version). Let $(\widehat{X}, \widehat{L})$ be a smooth threefold polarized by a very ample line bundle $\widehat{L}$. Assume that $(\widehat{X}, \widehat{L})$ is of log-general type. Let $(X, L)$ be the first reduction of $(\widehat{X}, \widehat{L})$. Further assume that the rational map associated to $K_{X}+L$ has a 1-dimensional image. Let $f=F \cap S$ be the transversal intersection of a general fiber $F$ of the connected part of $\varphi$ with a general smooth surface $S \in|L|$ as in (8). Then if $h^{0}\left(K_{X}+L\right) \geq 9$, the case $f \cdot f \geq 2$ does not occur.

Proof. Assume $f \cdot f \geq 2$. Let $h:=h^{0}\left(K_{X}+L\right)$. From (3.1) and (2.3) we have

$$
d_{1} \geq 6(h-1) ; \quad d_{2} \geq 2(h-1)^{2} ; \quad d_{3} \geq h-1 .
$$


Moreover, Miyaoka's inequality (5) and Tsuji's inequality (1.8) yield

$$
h-1 \geq \frac{h-1}{64}+\frac{h-1}{4}+\frac{2(h-1)^{2}}{18}-1 .
$$

Letting $x:=h-1$, we have $\frac{x^{2}}{9}+x\left(\frac{1}{64}+\frac{1}{4}-1\right)-\frac{17}{18} \leq 0$, or $64 x^{2}-423 x-544 \leq 0$. For $h \geq 9$, i.e., $x \geq 8$, the left term is positive, a contradiction.

Q.E.D.

We need the following lemma from $[5,(2.2)]$.

Lemma 3.3. Let $(\widehat{X}, \widehat{L})$ be a smooth threefold polarized by a very ample line bundle $\widehat{L}$. Assume that $\kappa(\widehat{X}) \geq 0$. Let $(X, L)$ be the first reduction of $(\widehat{X}, \widehat{L})$. If $d_{2}=d_{3}$, then $h^{0}\left(K_{X}+L\right) \geq h^{0}(\widehat{L})$.

Proof. It follows from the proof of Lemma (2.2) in [5].

Q.E.D.

We can now prove the main result of this section: a stronger version of the above result.

Theorem 3.4 (Refined version). Let $(\widehat{X}, \widehat{L})$ be a smooth threefold polarized by a very ample line bundle $\widehat{L}$. Assume that $(\widehat{X}, \widehat{L})$ is of log-general type. Let $(X, L)$ be the first reduction of $(\widehat{X}, \widehat{L})$. Further assume that the rational map associated to $K_{X}+L$ has a 1-dimensional image. Let $f=F \cap S$ be the transversal intersection of a general fiber $F$ of the connected part of $\varphi$ with a general smooth surface $S \in|L|$ as in (8). The following hold.

1) If $h^{0}\left(K_{X}+L\right) \geq 7$, then $f \cdot f \leq 1$ on $S$.

2) If $\kappa(\hat{X}) \geq 0$, then $f \cdot f \leq 1$ on $S$.

Proof. Assume $f \cdot f \geq 2$ and let $h:=h^{0}\left(K_{X}+L\right)$. By Proposition (3.2) we can assume $h \leq 8$. Now the following simple "C program," which we include for completeness (running for $7 \leq h \leq 8$ ) shows that there are no possible cases. The program contains all the relations stated in (3.1), the general bounds in (2.3) and (2.4), Miyaoka's inequality (4), Tsuji's inequality (1.8), Hodge index inequalities (2) and parity relations (3), and the double point formula inequalities of type (1.5) and (1.6). Note also that the inequality chi $:=\chi\left(\mathcal{O}_{S}\right) \leq 2 h-1$ follows from Tsuji's inequality, and that the bound $\gamma \leq d-9$, where $\gamma$ denotes the number of points blown up under the first reduction map, follows from $d:=L^{3} \geq \widehat{d}:=\widehat{L}^{3} \geq 9$ and the relation $\gamma=d-\widehat{d}($ see $(1.4))$. In the program $\mathrm{kf}:=\mathcal{K} \cdot f$, lf $:=L \cdot f, \mathrm{~d}:=L^{3}$, di $:=d_{i}$ for $i=1,2,3$, gamma $:=\gamma$, and ff $:=f \cdot f$ in $S$.

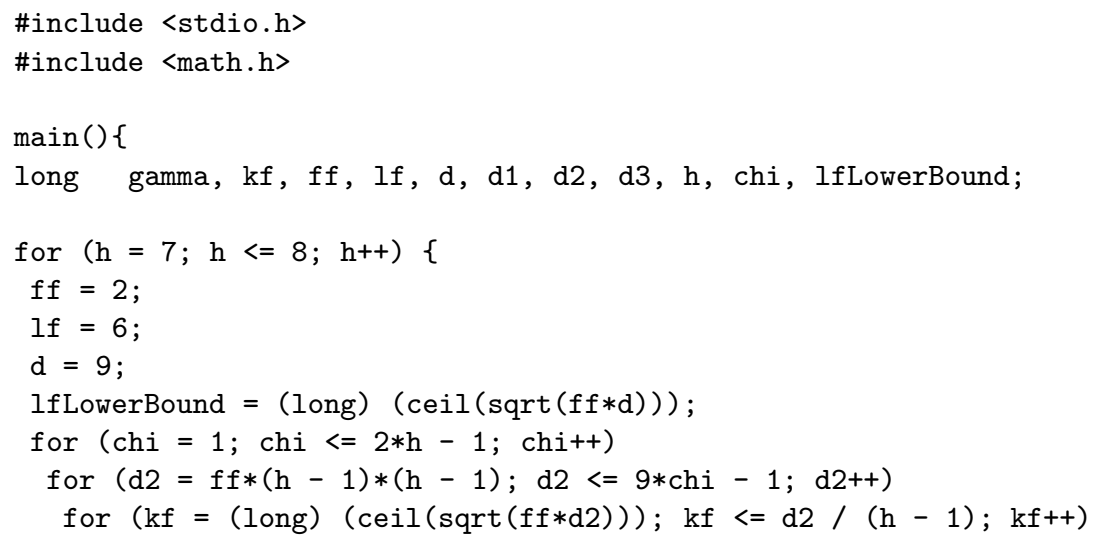




\section{\}\}\}}

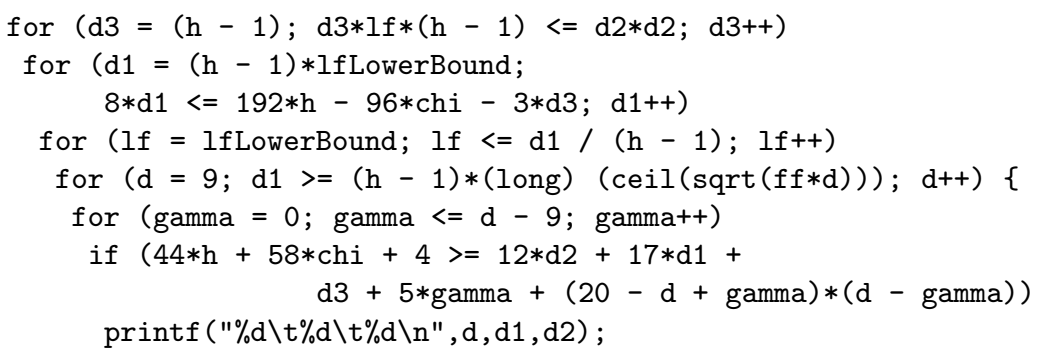

Assume now $\kappa(\widehat{X}) \geq 0$. Then from $[5,(3.3)]$ we know that $h^{0}\left(K_{X}+L\right) \geq 6$ unless $(\widehat{X}, \widehat{L})$ is a degree 5 hypersurface in $\mathbb{P}^{4}$. By [3, Theorem 4.4.1], $\widehat{X} \cong X$ and $K_{X}+L$ is very ample in this case. Thus we can assume $h^{0}\left(K_{X}+L\right) \geq 6$. Then exactly the same program as above (running now for $h=6$ ), with the relation $d_{3} \geq h-1$ replaced by the inequality $d_{3} \geq d_{2}$, shows that all possible cases have invariants $h=6, d_{2}=d_{3}=50$. Then Lemma (3.3) applies to rule out this case as soon as $h^{0}(\widehat{L}) \geq 7$. We insert here for completeness this new $C$ program (in the programs that follow we suppress the \#include lines which are identical to those in the above program).

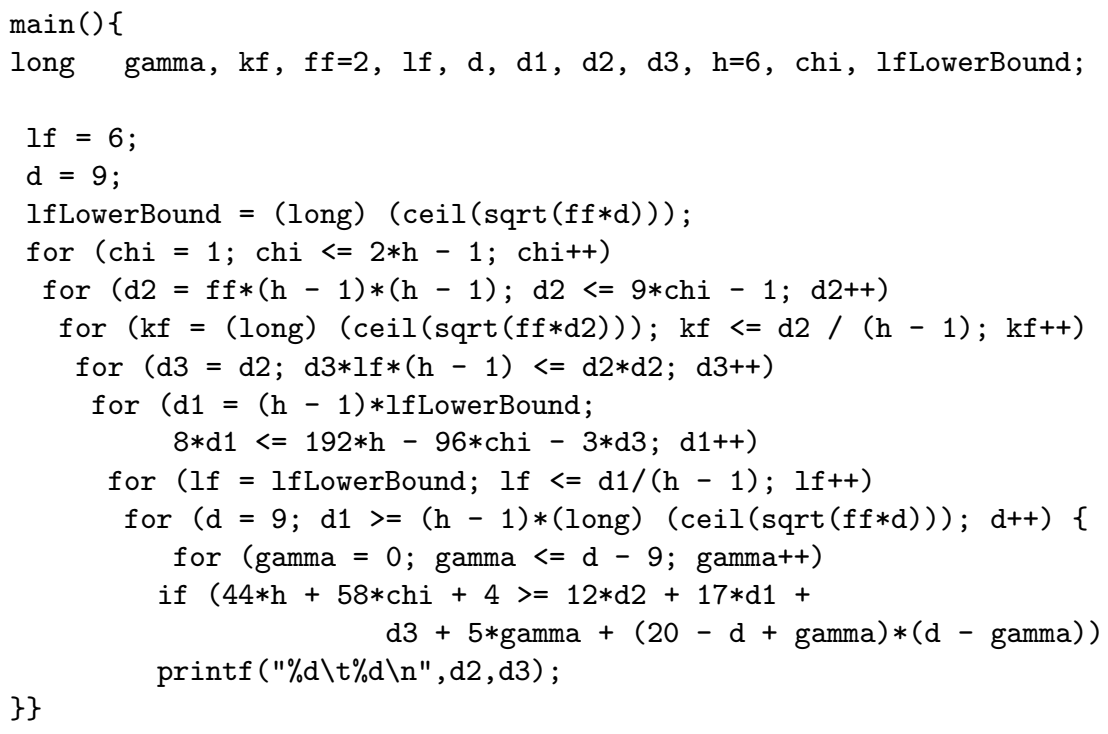

Since we may of course assume that $\Gamma(\widehat{L})$ embeds $\widehat{X}$ in $\mathbb{P}^{N}$ with $N \geq 5$, it remains to consider the case of threefolds in $\mathbb{P}^{5}$ of nonnegative Kodaira dimension. By [3, Theorem 4.4.1] we know that the first reduction map is an isomorphism in this case and hence that $\gamma=0$. Using this in the program above with the equality in the relation from (1.6), shows that no cases occur.

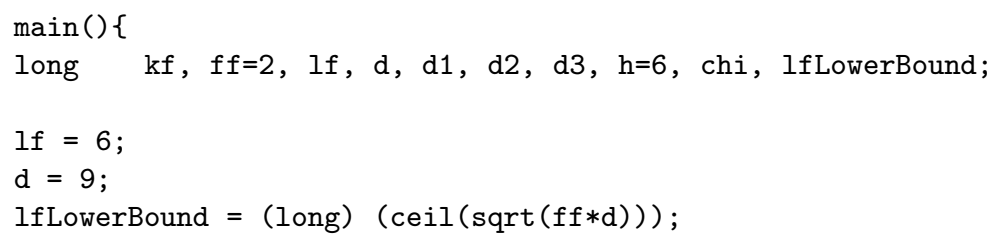




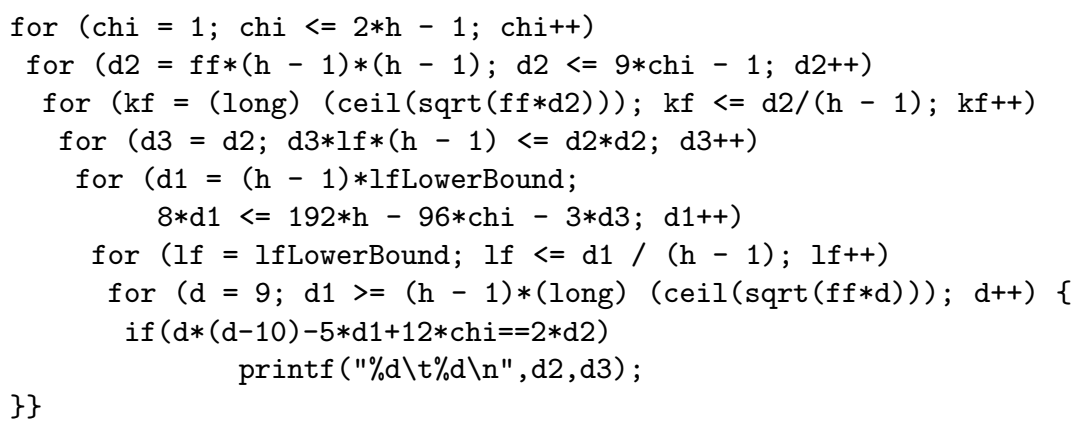

This completes the proof of the theorem.

Q.E.D.

\section{THE CASE $f \cdot f=1$}

Notation as in $\S 2$. By the results of $\S 3$ we can assume that the rational map $\varphi: X \rightarrow \Sigma$ associated to $\left|K_{X}+L\right|, \Sigma$ an irreducible curve, is not a morphism and that $f$ from (8) satisfies $f \cdot f:=(f \cdot f)_{S}=1$. By Lemma (2.2) we can assume without loss of generality that $r: X \rightarrow \mathbb{P}^{1}$, where $\varphi=s \circ r$ is the Remmert-Stein factorization of $\varphi$ in the sense of (2.1).

We need the following general fact.

Proposition 4.1. Let $(\widehat{X}, \widehat{L})$ be a smooth threefold polarized by a very ample line bundle $\widehat{L}$. Assume that $(\widehat{X}, \widehat{L})$ is of log-general type. Let $(X, L)$ be the first reduction of $(\widehat{X}, \widehat{L})$. Further assume that the rational map associated to $K_{X}+L$ has a 1 dimensional image. Let $F, F^{\prime}$ be distinct general fibers of the connected part of $\varphi$ as in (8), and let $\mathcal{K}:=K_{X}+L \approx t F+\mathcal{E}$ be as in (10), where $t:=h^{0}(\mathcal{K})-1$ and $\mathcal{E}$ is an effective divisor. We have:

1) $F$ is smooth;

2) $F \cap F^{\prime}$ is a line, i.e., $L \cdot F \cdot F=1$;

3) $F \cdot F \cdot F<0$ and $\mathcal{K} \cdot F \cdot F>0$;

4) $L \cdot L \cdot \mathcal{E} \geq \mathcal{E} \cdot F \cdot F \geq t+1$.

Proof. Let $f=F \cap S$ be the transversal intersection of $F$ with a general smooth surface $S \in|L|$. By Bertini's theorem we know that $F$ is smooth away from $F \cap F^{\prime}=\operatorname{Bs}\left|\mathcal{O}_{X}(F)\right|$. Let $\ell:=F \cap F^{\prime}$. One has

$$
L \cdot \ell=L \cdot F^{2}=f^{2}=1 .
$$

Then $\ell$ is a line. Hence in particular $\ell=F \cap F^{\prime}$ is smooth, and therefore the Cartier divisor $F$ is smooth in a neighborhood of $\ell$. Since by the above $F$ is smooth off $\ell$, we conclude that $F$ is smooth. This shows 1 ) and 2).

Since $\ell \cong \mathbb{P}^{1}$ the genus formula yields $\left(K_{X}+2 F\right) \cdot F \cdot F=-2$, and therefore, by $2),\left(K_{X}+L+2 F\right) \cdot F \cdot F=-2+L \cdot F \cdot F=-1$, or

$$
\mathcal{K} \cdot \ell+2 F \cdot \ell=-1 \text {. }
$$

Since $\mathcal{K}=K_{X}+L$ is nef we have $\mathcal{K} \cdot \ell \geq 0$ and therefore $F \cdot \ell=F \cdot F \cdot F^{\prime} \leq-1$. Hence $2 F \cdot \ell \leq-2$, so that $\mathcal{K} \cdot \ell>0$. This proves 3$)$.

Now compute $0<\mathcal{K} \cdot \ell=(t F+\mathcal{E}) \cdot F^{2}=t F^{3}+\mathcal{E} \cdot F^{2} \leq-t+\mathcal{E} \cdot \ell$. Thus $\mathcal{E} \cdot \ell \geq t+1$ and hence, by Lemma (1.11), we conclude that $L \cdot L \cdot \mathcal{E} \geq \mathcal{E} \cdot \ell \geq t+1$. This shows $4)$.

Q.E.D. 
4.2. General relations for $f \cdot f=1$. Let $d:=d_{0}, d_{1}, d_{2}, d_{3}$ be the pluridegrees of $(X, L)$ as in (1.4). Let $h:=h^{0}(\mathcal{K})$. Let $\mathcal{K}_{S}, L_{S}$ be the restrictions of $\mathcal{K}, L$ to a general smooth surface $S \in|L|$ respectively. Note that $\mathcal{K}_{S} \cong K_{S}, \mathcal{K} \cdot f=K_{S} \cdot f$, $L_{S} \cdot f=L \cdot f$. Let $g(f)$ be the arithmetic genus of $f$. Let $F, F^{\prime}$ be distinct general fibers of $r$. Recall that $\mathcal{K} \approx(h-1) F+\mathcal{E}$, with $\mathcal{E}$ an effective divisor, by (10). Then the following relations hold:

i) $L \cdot f \geq\lceil\sqrt{d}\rceil$;

ii) $d_{1} \geq L \cdot f(h-1)+h$;

iii) $d_{2} \geq(h-1)(2 h-1)$;

iv) $d_{3} \geq(h-1)^{2}$;

v) $\left\lceil\sqrt{d_{2}}\right\rceil \leq \mathcal{K} \cdot f \leq d_{2} /(h-1)$;

vi) $2 g(f)-2=\mathcal{K} \cdot f+1$.

Relations i), v), vi) are proved exactly as in (3.1) by putting $f \cdot f=1$. Let $F$ be a general fiber of the connected part of the rational map $\varphi$ as in (8).

ii) We have, by using (4.1), 4),

$$
d_{1}=L \cdot L \cdot \mathcal{K}=(h-1) L \cdot L \cdot F+L \cdot L \cdot \mathcal{E} \geq(h-1) L \cdot f+h .
$$

iii) Let $t:=h-1, \ell:=F \cap F^{\prime}$, and compute

$$
\mathcal{K} \cdot F \cdot L=\mathcal{K}_{F} \cdot L_{F}=\left(t F_{F}+\mathcal{E}_{F}\right) \cdot L_{F}=t \ell \cdot L_{F}+\mathcal{E}_{F} \cdot L_{F} .
$$

Since $\ell \cdot L_{F}=1$, by $\left.(4.1), 2\right)$ we get

$$
\mathcal{K} \cdot F \cdot L=t+\mathcal{E}_{F} \cdot L_{F},
$$

and by Lemma (1.11) applied to $F, L_{F}-\ell$ is nef. Therefore, using (4.1), 4), $\mathcal{E}_{F} \cdot L_{F}=(L-\ell)_{F} \cdot \mathcal{E}_{F}+\ell \cdot \mathcal{E}_{F} \geq \ell \cdot \mathcal{E}_{F}=\mathcal{E} \cdot F \cdot F \geq t+1$. Thus, by (11), we conclude $\mathcal{K} \cdot F \cdot L \geq 2 t+1$. Now

$$
d_{2}=\mathcal{K} \cdot \mathcal{K} \cdot L=t F \cdot \mathcal{K} \cdot L+\mathcal{E} \cdot \mathcal{K} \cdot L .
$$

Since $\mathcal{E} \cdot \mathcal{K} \cdot L \geq 0$ we find $d_{2} \geq t(2 t+1)$, or $d_{2} \geq(h-1)(2 h-1)$.

iv) Let $t:=h-1$. Since $\mathcal{K} \cdot \mathcal{K} \cdot \mathcal{E} \geq 0, \mathcal{K} \cdot \mathcal{E} \cdot F \geq 0$ we have

$$
d_{3}=\mathcal{K}^{3}=\mathcal{K}^{2} \cdot(t F+\mathcal{E}) \geq t \mathcal{K}^{2} \cdot F=t \mathcal{K} \cdot(t F+\mathcal{E}) \cdot F \geq t^{2} \mathcal{K} \cdot F \cdot F .
$$

Since $\mathcal{K} \cdot F \cdot F>0$ by $(4.1), 3)$, we get $d_{3} \geq t^{2}$, or $d_{3} \geq(h-1)^{2}$.

Q.E.D.

As in the previous section we need to prove a "coarse" preparatory result which states that for $h:=h^{0}\left(K_{X}+L\right) \geq 8$, the case $f \cdot f=1$ does not occur.

Proposition 4.3 (Coarse version). Let $(\widehat{X}, \widehat{L})$ be a smooth threefold polarized by a very ample line bundle $\widehat{L}$. Assume that $(\widehat{X}, \widehat{L})$ is of log-general type. Let $(X, L)$ be the first reduction of $(\widehat{X}, \widehat{L})$. Further assume that the rational map associated to $K_{X}+L$ has a 1-dimensional image. Let $f=F \cap S$ be the transversal intersection of a general fiber $F$ of the connected part of $\varphi$ with a general smooth surface $S \in|L|$ as in (8). Then if $h^{0}\left(K_{X}+L\right) \geq 8$, the case $f \cdot f=1$ does not occur.

Proof. Assume $f \cdot f=1$. Let $h:=h^{0}\left(K_{X}+L\right)$. From (2.3) and (4.2) we have

$$
d_{1} \geq 4(h-1)+h=5(h-1)+1 ; \quad d_{2} \geq(h-1)(2 h-1) ; \quad d_{3} \geq(h-1)^{2} .
$$

Therefore Miyaoka's inequality (5) and Tsuji's inequality (1.8) yield

$$
h-1 \geq \frac{(h-1)^{2}}{64}+\frac{5(h-1)+1}{24}+\frac{(2 h-1)(h-1)+1}{18}-1 .
$$


Letting $x:=h-1$, we have $x \geq \frac{x^{2}}{64}+\frac{5 x+1}{24}+\frac{2 x^{2}+x+1}{18}-1$, which gives $73 x^{2}-424 x-$ $550 \leq 0$. For $h \geq 8$, i.e., $x \geq 7$, the left term is positive, so we get a contradiction. Q.E.D.

We can now prove a stronger version of the result above.

Theorem 4.4 (Refined version). Let $(\widehat{X}, \widehat{L})$ be a smooth threefold polarized by a very ample line bundle $\widehat{L}$. Assume that $(\widehat{X}, \widehat{L})$ is of log-general type. Let $(X, L)$ be the first reduction of $(\widehat{X}, \widehat{L})$. Further assume that the rational map associated to $K_{X}+L$ has a 1-dimensional image. Let $f=F \cap S$ be the transversal intersection of a general fiber $F$ of the connected part of $\varphi$ with a general smooth surface $S \in|L|$ as in (8). If $h^{0}\left(K_{X}+L\right) \geq 5$, then the case $f \cdot f=1$ does not occur.

Proof. Assume $f \cdot f=1$. Let $h:=h^{0}\left(K_{X}+L\right)$. By Proposition (4.3) we can assume $h \leq 7$. Now the following simple "C program," which we include for completeness (running for $5 \leq h \leq 7$ ), shows that there are no possible cases. The program contains the relations contained in (4.2), the general bounds (2.3) and (2.4), Miyaoka's inequality (4), Tsuji's inequality (1.8), the Hodge index inequalities (2), and the parity relations (3). Note also that the inequality chi $:=\chi\left(\mathcal{O}_{S}\right) \leq 2 h-1$ follows from Tsuji's inequality. In the program $\mathrm{kf}:=\mathcal{K} \cdot f$, lf $:=L \cdot f, \mathrm{~d}:=L^{3}$, $\mathrm{di}:=d_{i}$ for $i=1,2,3$.

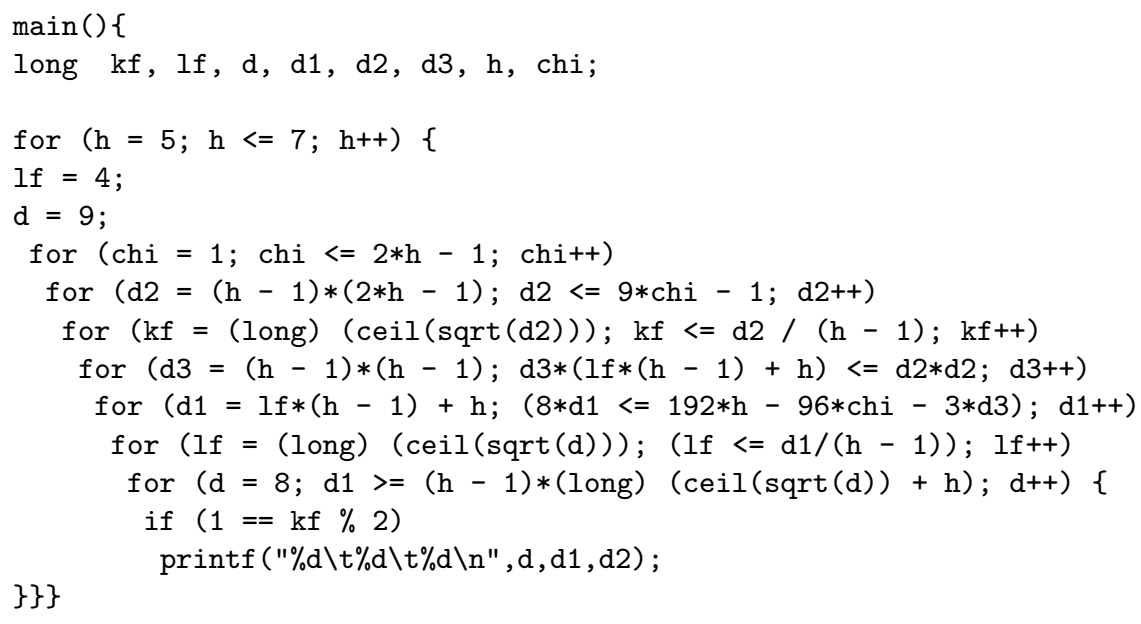

Q.E.D.

As an immediate consequence of the results above we can now prove the first main result of the paper.

Theorem 4.5. Let $(\widehat{X}, \widehat{L})$ be a smooth threefold polarized by a very ample line bundle $\widehat{L}$. Assume that $(\widehat{X}, \widehat{L})$ is of log-general type. Let $(X, L)$ be the first reduction of $(\widehat{X}, \widehat{L})$. Further assume that the rational map, $\varphi$, associated to $K_{X}+L$ has a 1-dimensional image. Let $r: X \rightarrow C$ be the part of the Remmert-Stein factorization with connected fibers $($ see $(2.1))$. If either $h^{0}\left(K_{X}+L\right) \geq 7$ or $\kappa(\widehat{X}) \geq 0$ or $g(C)>0$, then $\varphi$ is a morphism.

Proof. Lemma (2.2) gives the result if $g(C)>0$. Let $f=F \cap S$ be the transversal intersection of a general fiber $F$ of the connected part of $\varphi$ with a general smooth surface $S \in|L|$ as in (8). By combining Theorems (3.4) and (4.4) we conclude that 
$f \cdot f=0$ on $S$ if either $h^{0}\left(K_{X}+L\right) \geq 7$ or $h^{0}\left(K_{X}+L\right) \geq 6$ and $\kappa(\widehat{X}) \geq 0$. From $[5,(3.3)]$ we know that, if $\kappa(\widehat{X}) \geq 0, h^{0}\left(K_{X}+L\right) \geq 6$ unless $(\widehat{X}, \widehat{L})$ is a degree 5 hypersurface of $\mathbb{P}^{4}$. In this last case, by [3, 4.4.1], $\widehat{X} \cong X$ and $K_{X}+L$ is very ample.

Q.E.D.

\section{The CASE $f \cdot f=0$ (THE MORPhism CASE)}

We use the notation as in $\S 2$. Theorem (4.5) gives criteria for the rational map $\varphi: X \rightarrow \Sigma$ associated to $\left|K_{X}+L\right|, \Sigma$ an irreducible curve, to be a morphism. In this section and in $\S 6$ we will study the structure of such a morphism $\varphi$.

5.1. General relations for $f \cdot f=0$. Let $d:=d_{0}, d_{1}, d_{2}, d_{3}$ be the pluridegrees of $(X, L)$ as in (1.4). Let $h:=h^{0}(\mathcal{K}), \mathcal{K}=K_{X}+L$. Let $F$ be a general fiber of the connected part of the morphism $\varphi$ and let $f=F \cap S$ be the transversal intersection of $F$ with a general smooth surface $S \in|L|$. Recall that $\mathcal{K} \approx t F+\mathcal{E}$, with $\mathcal{E}$ an effective divisor in the base locus of $|\mathcal{K}|$, as in (10). Since $h^{0}(\mathcal{K})=h$, we see that $t \geq h-1$, with equality if and only if $C$ in (2.1) is rational. The following relations hold:

i) $L \cdot L \cdot \mathcal{E} \geq 2$;

ii) $d_{1} \geq(h-1) L \cdot L \cdot F+2$;

iii) $d_{2} \geq(h-1)(2 g(f)-2)$;

iv) $d_{3} \geq(h-1)(g(f)-2)$.

i) We have $L \cdot L \cdot \mathcal{E} \geq 0$. Assume $L \cdot L \cdot \mathcal{E}=1$. Then $\mathcal{E}$ is a linear $\mathbb{P}^{2}$ and therefore $\varphi(\mathcal{E})$ is a point, so that $\mathcal{E}$ is contained in a fiber, $F$. Thus the cycles $F \cdot \mathbb{P}^{2}, F \cdot \mathcal{E}$ are numerically trivial, and hence $\mathcal{E} \cdot \mathcal{E} \cdot F=0$. Since $\varphi$ is a morphism, $F \cdot F$ is numerically trivial. Moreover $\mathcal{K} \cdot \mathcal{K} \cdot F>0$, since $\mathcal{K}$ is nef and big and $F$ moves (see, e.g., $[6,(2.1 .2)])$. Therefore we get the contradiction

$$
0<\mathcal{K} \cdot \mathcal{K} \cdot F=\left(t^{2} F \cdot F+2 t F \cdot \mathcal{E}+\mathcal{E} \cdot \mathcal{E}\right) \cdot F=\mathcal{E} \cdot \mathcal{E} \cdot F=0 .
$$

ii) By using i) we have

$$
d_{1}=\mathcal{K} \cdot L \cdot L \geq(h-1) L \cdot L \cdot F+L \cdot L \cdot \mathcal{E} \geq(h-1) L \cdot L \cdot F+2 .
$$

iii) We have $\mathcal{K} \cdot L \cdot \mathcal{E} \geq 0$ and, by the genus formula,

$$
\mathcal{K} \cdot L \cdot F=\left(K_{F}+L_{F}\right) \cdot L_{F}=2 g(f)-2 .
$$

Thus compute

$$
d_{2}=\mathcal{K} \cdot \mathcal{K} \cdot L=\mathcal{K} \cdot L \cdot(t F+\mathcal{E}) \geq(h-1) \mathcal{K} \cdot L \cdot F=(h-1)(2 g(f)-2) .
$$

iv) Note that since $L$ is very ample off a finite set of points, we can assume that $L_{F}$ is spanned, and in fact very ample, on the general fiber $F$. Therefore, since $K_{X}+L$ is nef and big, from adjunction theory for surfaces (see e.g., $[6,(9.2 .3)]$ ) we can assume that $K_{F}+L_{F}$ is spanned and big. Let $A$ be a general element in $\left|K_{F}+L_{F}\right|$. Then from the exact sequence

$$
0 \rightarrow \mathcal{O}_{F}(-A) \rightarrow \mathcal{O}_{F} \rightarrow \mathcal{O}_{A} \rightarrow 0
$$

we get $q(F) \leq h^{1}\left(\mathcal{O}_{A}\right)=g\left(K_{F}+L_{F}\right)$. Hence $2 g\left(K_{F}+L_{F}\right)-2 \geq 2 q(F)-2$, or $2\left(K_{F}+L_{F}\right)^{2}-L_{F} \cdot\left(K_{F}+L_{F}\right) \geq 2 q(F)-2$, or since $\left(K_{F}+L_{F}\right) \cdot L_{F}=2 g(f)-2$,

$$
\left(K_{F}+L_{F}\right)^{2} \geq g(f)+q(F)-2 \geq g(f)-2 .
$$


Thus

$$
d_{3}=\mathcal{K}^{3} \geq(h-1) \mathcal{K}^{2} \cdot F=(h-1)\left(K_{F}+L_{F}\right)^{2} \geq(h-1)(g(f)-2) .
$$

We can now prove the following boundedness result.

Theorem 5.2. Let $(\widehat{X}, \widehat{L})$ be a smooth threefold polarized by a very ample line bundle $\widehat{L}$. Assume that $(\widehat{X}, \widehat{L})$ is of log-general type. Let $(X, L)$ be the first reduction of $(\widehat{X}, \widehat{L})$. Further assume that the rational map associated to $K_{X}+L$ has a 1 dimensional image. Let $f=F \cap S$ be the transversal intersection of a general fiber $F$ of the connected part of $\varphi$ with a general smooth surface $S \in|L|$ as in (8). Assume that $\varphi$ is a morphism (e.g., see (4.5)). Then $g(f) \leq 10$. Furthermore:

1) if $h^{0}\left(K_{X}+L\right) \geq 6$ then $g(f) \leq 7$;

2) if $h^{0}\left(K_{X}+L\right) \geq 10$ then $g(f) \leq 6$.

Proof. Since $L$ is very ample off a finite set, we can assume without loss of generality that $L_{F}$ is very ample for a general fiber $F$.

If $g(f) \geq 11$ then we can assume by Castelnuovo's bound for the genus that $\delta:=L \cdot f \geq 7$. Let $h:=h^{0}\left(K_{X}+L\right)$. Miyaoka's inequality (5), Tsuji's inequality (1.8), and the relations ii), iii), and iv) above give

$$
h \geq \frac{9(h-1)}{64}+\frac{7(h-1)}{24}+\frac{1}{12}+\frac{10(h-1)}{9}+\frac{1}{18} .
$$

It is easy to see that the inequality above is of the type

$$
c h \leq c^{\prime},
$$

where $c, c^{\prime}$ are positive constants. Therefore whenever it leads to a contradiction for a given value $h_{0}$ of $h$, then we get a contradiction for each $h \geq h_{0}$. We see that it leads to a contradiction if $h \geq 3$. If $h=2$ we obtain a contradiction using Miyaoka's inequality (6), taking the integrality into account. Thus $g(f) \leq 10$.

We claim that if $g(f) \geq 7$ then $h^{0}\left(L_{f}\right) \geq 4$. Indeed, if this is false then $f$ would be a plane curve, and hence we know that $L \cdot f \geq 6$ with $g(f) \geq 10$. Let $g:=g(f)$. Thus using the inequalities in (5.1), the assumption $\delta \geq 6$, Miyaoka's inequality (5), and Tsuji's inequality (1.8) yields

$$
\begin{aligned}
h & \geq \frac{(h-1)(g-2)}{64}+\frac{h-1}{4}+\frac{1}{12}+\frac{(h-1)(2 g-2)}{18}+\frac{1}{18} \\
& \geq \frac{h-1}{8}+\frac{h-1}{4}+\frac{1}{12}+h-1+\frac{1}{18} .
\end{aligned}
$$

Again this inequality is of the type $c h \leq c^{\prime}$, where $c, c^{\prime}$ are positive constants. Therefore whenever (14) leads to a contradiction for a given value $h_{0}$ of $h$, then we get a contradiction for each $h \geq h_{0}$. We see that it leads to a contradiction if $h \geq 4$.

Assume $g:=g(f) \geq 7$. We claim that

$$
L \cdot L \cdot F \geq 8
$$

By the last paragraph we can assume that $h^{0}\left(L_{f}\right) \geq 4$. Thus we see that $f$ is a curve of degree $\delta=L \cdot f$ in $\mathbb{P}^{N}$, with $N \geq 3$. Using Castelnuovo's inequality shows that $\delta \leq 7$ implies the contradiction $g \leq 6$. Hence, by combining (5.1), ii) and (15), 
we get $d_{1} \geq 8(h-1)+2$. Using the inequalities in (5.1), Miyaoka's inequality (5), and Tsuji's inequality (1.8) yields

$$
h \geq \frac{(h-1)(g-2)}{64}+\frac{h-1}{3}+\frac{1}{12}+\frac{(h-1)(2 g-2)}{18}+\frac{1}{18} .
$$

As before this inequality is of the type $c h \leq c^{\prime}$, where $c, c^{\prime}$ are positive constants. Therefore whenever (16) leads to a contradiction for a given value $h_{0}$ of $h$, then we get a contradiction for each $h \geq h_{0}$.

If $g \geq 8$ then (16) becomes

$$
h \geq \frac{3(h-1)}{32}+\frac{h-1}{3}+\frac{1}{12}+\frac{7(h-1)}{9}+\frac{1}{18} .
$$

A direct computation shows that for $h \geq 6$, this yields a contradiction. Thus (5.2), 1) is proved.

To prove (5.2), 2), assume $g \geq 7$. Then inequality (16) is still true and, arguing as above, it is enough to check that we have a contradiction for $h=10$. Use the integrality relation (see (6))

$$
\chi\left(\mathcal{O}_{S}\right) \geq\left\lceil\frac{d_{2}+1}{9}\right\rceil \geq\left\lceil\frac{(h-1)(2 g-2)+1}{9}\right\rceil,
$$

which, for $h=10, g \geq 7$, gives $\frac{\chi\left(\mathcal{O}_{S}\right)}{2} \geq\left\lceil\frac{109}{9}\right\rceil / 2=\frac{13}{2}$. Hence (16) leads to the contradiction $10 \geq \frac{45}{64}+3+\frac{1}{12}+\frac{13}{2}=10.2$. Since we used integrality, we need to proceed further. Indeed, to conclude the proof we have to find the first value of $h \geq 10$ such that (16) leads to a contradiction without using the integrality trick above.

For $h=11,12$, integrality again gives a contradiction. For $h=13$, (16) gives the contradiction $13 \geq \frac{15}{16}+4+\frac{1}{12}+8+\frac{1}{18}=13.6$ Q.E.D.

\section{The STRUCTURE OF THE SECOND ADJUNCTION MAPPING IN THE CASE OF NONNEGATIVE KODAIRA DIMENSION}

Notation as in $\S 2$. By Theorem (4.5) we know that the rational map $\varphi:=X \rightarrow \Sigma$ associated to $\left|K_{X}+L\right|, \Sigma$ an irreducible curve, is always a morphism if $\kappa(X) \geq 0$. In this section we study the structure of the morphism $\varphi$. We prove the following second main result of the paper.

Theorem 6.1. Let $(\widehat{X}, \widehat{L})$ be a smooth threefold polarized by a very ample line bundle $\widehat{L}$. Assume that $\kappa(\widehat{X}) \geq 0$. Let $(X, L)$ be the first reduction of $(\widehat{X}, \widehat{L})$. Further assume that the rational map $\varphi$ associated to $K_{X}+L$ has a 1-dimensional image. Let $f=F \cap S$ be the transversal intersection of a general fiber $F$ of the connected part $r$ of $\varphi$ with a general smooth surface $S \in|L|$ as in (8). Then $\varphi$ is a morphism and

1) $g(f) \leq 6$;

2) for $h:=h^{0}\left(K_{X}+L\right) \gg 0($ e.g., $h \geq 21)$ we have $g(f) \leq 5, F$ is either a $K 3$ surface or the blowing up at one point of a $K 3$ surface, and $C:=r(X)$ is a curve of genus $g(C) \leq 1$. Precisely, the following cases are possible (in each case the embedding is given by $\left.\Gamma\left(L_{F}\right)\right)$ :

i) $g(f)=3, L \cdot f=4, F$ is a quartic in $\mathbb{P}^{3}$;

ii) $g(f)=4, L \cdot f=6, F$ is the complete intersection of a quadric and a cubic hypersurface in $\mathbb{P}^{4}$; 
iii) $g(f)=5, L \cdot f=8, F$ is the complete intersection of three quadrics in $\mathbb{P}^{5}$

iv) $g(f)=5, L \cdot f=7, F$ is embedded in $\mathbb{P}^{4}$ and the reduction of $\left(F, L_{F}\right)$ is as in case iii).

Proof. By (4.5) (see also $[5,(3.3)]$ ) we know that $h \geq 6$ and that $\varphi$ is a morphism. Assume $g:=g(f) \geq 7$. By $(5.2), 1)$, and its proof, we know that $g=7$ and $\delta:=L \cdot f \geq 8$. By virtue of the relations

$$
\begin{aligned}
& d_{1} \geq(h-1) L \cdot f+2 \geq 8(h-1)+2, \\
& d_{3} \geq d_{2} \geq(2 g-2)(h-1) \geq 12(h-1)
\end{aligned}
$$

from (5.1) and (1.10), and Miyaoka's inequality (5), Tsuji's inequality (1.8) yields

$$
h \geq \frac{3(h-1)}{16}+\frac{h-1}{3}+\frac{1}{12}+\frac{2(h-1)}{3}+\frac{1}{18} .
$$

This gives the numerical contradiction $\frac{15}{16} \leq \frac{3(h-1)}{16} \leq \frac{31}{36}$.

To prove (6.1), 2) we can assume $g=6$. First consider the case when $F \subset \mathbb{P}^{N}$, with $N \geq 4$, so that $f \subset \mathbb{P}^{r}$ with $r \geq 3$. Note that we can also assume $L \cdot f \geq 7$, since otherwise Castelnuovo's bound (1.9) would imply $g \leq \operatorname{Castel}(6,3)=4$, and we would be done.

Assume $\delta=L \cdot f=7$. Then by Castelnuovo's bound $F$ lies in $\mathbb{P}^{4}$, and Lemma (1.6) gives

$$
K_{F} \cdot K_{F}=\chi\left(\mathcal{O}_{F}\right)-18 .
$$

Since $K_{F}=\mathcal{K}_{F}-L_{F}$, and by noting that $\mathcal{K} \cdot F \cdot L=\left(K_{F}+L_{F}\right) \cdot L_{F}=2 g(f)-2=10$ and $L \cdot L \cdot F=\delta=7$, we also have

$$
K_{F} \cdot K_{F}=\mathcal{K} \cdot \mathcal{K} \cdot F-2 \mathcal{K} \cdot F \cdot L+L \cdot L \cdot F=\mathcal{K} \cdot \mathcal{K} \cdot F-13 .
$$

Therefore, by (18),

$$
\mathcal{K}_{F} \cdot \mathcal{K}_{F}=6 \chi\left(\mathcal{O}_{F}\right)-5
$$

so that, recalling inequality (12), we get $6 \chi\left(\mathcal{O}_{F}\right)-5 \geq 4$, or $\chi\left(\mathcal{O}_{F}\right) \geq 2$. This implies $\kappa(F) \geq 0$, and therefore $\mathcal{K}_{F} \cdot \mathcal{K}_{F} \geq 2\left(\chi\left(\mathcal{O}_{F}\right)+g(f)-3\right) \geq 10$ by $[6$, (10.1.2), 2)]. By using again (19) we find $\chi\left(\mathcal{O}_{F}\right) \geq 3$, and hence $\mathcal{K}_{F} \cdot \mathcal{K}_{F} \geq 13$.

From inequality $(13)$ we conclude that $d_{3} \geq 13(h-1) \geq 65$. Furthermore relations (5.1) yield $d_{2} \geq 10(h-1) \geq 50, d_{1} \geq 7(h-1)+2 \geq 37$. Thus Tsuji's inequality (1.8) gives $\chi\left(\mathcal{O}_{S}\right) \leq 6$, and hence the double point formula (1.5) leads to $(d-20) d \geq 678$, or $d \geq 38, d=L^{3}$. Therefore the Hodge index relation $d_{1}^{2} \geq d d_{2} \geq 1900$ yields $d_{1} \geq 44$, and hence $d_{2}^{2} \geq d_{1} d_{3} \geq 2860$ implies $d_{2} \geq 54$. This contradicts Miyaoka's inequality $d_{2}<9 \chi\left(\mathcal{O}_{S}\right) \leq 54$.

Thus we conclude that $\delta=L \cdot f \geq 8$. Then Tsuji's inequality (1.8) gives

$$
h \geq \frac{5(h-1)}{32}+\frac{8(h-1)}{24}+\frac{1}{12}+\frac{5(h-1)}{9}+\frac{1}{18} .
$$

Writing $c:=\frac{5}{32}+\frac{8}{24}+\frac{5}{9}$, we have $c h \leq h+c-\frac{1}{12}-\frac{1}{18}$. Since $c>1$, we see that this leads to a contradiction for $h \geq 21$.

Consider now the case when $F \subset \mathbb{P}^{3}$. Then $f$ is a plane curve and, since we are assuming $g=6$, we have $L \cdot f=5$. Therefore $K_{F} \cong \mathcal{O}_{F}(1)$ and hence $K_{F}+L_{F} \cong$ 
$\mathcal{O}_{F}(2)$. Thus $\mathcal{K} \cdot \mathcal{K} \cdot F=\left(K_{F}+L_{F}\right)^{2}=20$. Then, by looking over the proof of (5.1), iv), we conclude that

$$
d_{3} \geq \mathcal{K} \cdot \mathcal{K} \cdot F(h-1) \geq 20(h-1)
$$

in this case. Therefore exactly the same argument as in the previous case, using (20) and $L \cdot f=5$, gives now

$$
h \geq \frac{5(h-1)}{16}+\frac{5(h-1)}{24}+\frac{1}{12}+\frac{5(h-1)}{9}+\frac{1}{18} ;
$$

or, writing $c^{\prime}:=\frac{5}{16}+\frac{5}{24}+\frac{5}{9}$, we obtain $c^{\prime} h \leq h+c^{\prime}-\frac{1}{12}-\frac{1}{18}$. Since $c^{\prime}>1$, we see that this leads to a contradiction for $h \geq 21$. Thus we conclude that $g(f) \leq 5$ if $h \geq 21$.

Let us prove that the general fiber $F$ of $\varphi$ is a $K 3$ surface if $g \leq 5$. We refer, e.g., to $[6, \S \S 8.9,10.2,11.6]$ for the well known classification of polarized pairs of sectional genus $\leq 4$ that we use in the sequel. We also use the well known additivity formula for Kodaira dimension, i.e., $\kappa(X) \leq \kappa(F)+1$.

First note that $g \geq 3$. Indeed, otherwise $F$ would be ruled and hence we would have the contradiction $\kappa(X)<0$.

If $g=3$, the only possible case is $L \cdot f=4$ and $F$ is a quartic surface in $\mathbb{P}^{3}$. If $g=4$, the only possible case is $L \cdot f=6$ and $F$ is the complete intersection of a quadric and a cubic in $\mathbb{P}^{5}$. Note that in both these cases $F$ is a $K 3$ surface, so we are as in 2), i), ii).

Thus we can assume $g=5$. We claim that $7 \leq \delta:=L \cdot f \leq 8$. To see this note that since $F$ is of nonnegative Kodaira dimension, then $t K_{F}$ is effective for some positive integer $t$ and therefore $L_{F} \cdot K_{F} \geq 0$. Hence the genus formula yields $L \cdot f \leq 2 g-2=8$. Moreover $g=5$ implies that $\left|L_{F}\right|$ embeds $F$ in $\mathbb{P}^{N}$ with $N \geq 4$, since otherwise $(F, f)$ would be a polarized surface of sectional genus 5 in $\mathbb{P}^{3}$, which is not possible. Then Castelnuovo's bound gives $L \cdot f \geq 7$, since otherwise we would have the contradiction $5=g \leq \operatorname{Castel}(6,3)=4$.

Note that in both the cases $\delta=L \cdot f=7,8$ one has $\delta \leq 2 g$, so the usual Clifford's theorem applies. Let $L \cdot f=7$. Then by Clifford's theorem, $h^{0}\left(L_{f}\right) \leq \frac{\delta}{2}+1$, or $h^{0}\left(L_{f}\right) \leq 4$. Since there are no curves in $\mathbb{P}^{2}$ of degree 7 and genus 5 , we conclude that $h^{0}\left(L_{f}\right)=4$ and $F$ lies in $\mathbb{P}^{4}$. Therefore Lemma (1.6) gives

$$
6 \chi\left(\mathcal{O}_{F}\right)-13=K_{F}^{2} \text {. }
$$

The genus formula yields $K_{F} \cdot L_{F}=2 g-2-L \cdot f=1$, and hence from the Hodge index relation $7 K_{F}^{2}=L_{F}^{2} K_{F}^{2} \leq\left(K_{F} \cdot L_{F}\right)^{2}=1$ we obtain $K_{F}^{2} \leq 0$. Therefore, by $(21)$

$$
\chi\left(\mathcal{O}_{F}\right) \leq 2 .
$$

Since $K_{F}^{2}+2 K_{F} \cdot L_{F}+L \cdot f=K_{F}^{2}+4 g-4-\delta$, we have from (21)

$$
\left(K_{F}+L_{F}\right)^{2}=K_{F}^{2}+9=6 \chi\left(\mathcal{O}_{F}\right)-4 .
$$

On the other hand the general inequality

$$
\left(K_{F}+L_{F}\right)^{2} \geq 2\left(\chi\left(\mathcal{O}_{F}\right)+g-3\right)=2 \chi\left(\mathcal{O}_{F}\right)+4
$$

holds true (see $[6,(10.1 .2 .2)]$ ). It thus follows that $2 \chi\left(\mathcal{O}_{F}\right)+4 \leq 6 \chi\left(\mathcal{O}_{F}\right)-4$, or $\chi\left(\mathcal{O}_{F}\right) \geq 2$. Therefore, by $(22), \chi\left(\mathcal{O}_{F}\right)=2$. Hence in particular $h^{0}\left(K_{F}\right) \geq 1$. Note also that we can assume $L_{F}$ very ample on the general fiber $F$. Recall that $K_{F} \cdot L_{F}=1$. Then there exists a line $\ell \in\left|K_{F}\right|$ such that $\ell^{2}=-1$. Let $\pi: F \rightarrow F^{\prime}$ 
be the contraction of $\ell$. Hence $K_{F} \approx \pi^{*} K_{F^{\prime}}+\ell$, so that $\pi^{*} K_{F^{\prime}} \approx \mathcal{O}_{F}$ and hence $K_{F^{\prime}} \approx \mathcal{O}_{F^{\prime}}$. Thus $p_{g}\left(F^{\prime}\right)=1$ and, since $\chi\left(\mathcal{O}_{F}\right)=\chi\left(\mathcal{O}_{F^{\prime}}\right)=2$, we conclude that $q\left(F^{\prime}\right)=0$. This means that $F^{\prime}$ is a $K 3$ surface and $F$ is a blowing up at one point of it.

Let $L \cdot f=8$. Then by Clifford's theorem $h^{0}\left(L_{f}\right) \leq 5$. Since there are no curves $f \subset \mathbb{P}^{2}$ of degree 8 and genus 5 , we conclude that $4 \leq h^{0}\left(L_{f}\right) \leq 5$. Let $h^{0}\left(L_{f}\right)=4$. Then $F$ lies in $\mathbb{P}^{4}$, and Lemma (1.6) gives

$$
6 \chi\left(\mathcal{O}_{F}\right)-8=K_{F}^{2}
$$

The same arguments as in the previous case $L \cdot f=7$ now give us

$$
\left(K_{F}+L_{F}\right)^{2}=K_{F}^{2}+8=6 \chi\left(\mathcal{O}_{F}\right)
$$

and, recalling $[6,(10.1 .2 .2)]$,

$$
\left(K_{F}+L_{F}\right)^{2} \geq 2 \chi\left(\mathcal{O}_{F}\right)+4
$$

It thus follows that $2 \chi\left(\mathcal{O}_{F}\right)+4 \leq 6 \chi\left(\mathcal{O}_{F}\right)$, or $\chi\left(\mathcal{O}_{F}\right) \geq 1$.

The genus formula yields $K_{F} \cdot L_{F}=0$, and hence from the Hodge index relation $8 K_{F}^{2}=L_{F}^{2} K_{F}^{2} \leq\left(K_{F} \cdot L_{F}\right)^{2}=0$ we get $K_{F}^{2} \leq 0$. Then, by $(23), \chi\left(\mathcal{O}_{F}\right) \leq 1$. Thus $\chi\left(\mathcal{O}_{F}\right)=1$ and $K_{F}^{2}=-2$. Hence in particular equality holds in (24). Then from $[6,(10.1 .2 .2)]$ we know that the reduction $Y$ of $F$ is a $K 3$ surface. Therefore we find the contradiction $\chi\left(\mathcal{O}_{Y}\right)=\chi\left(\mathcal{O}_{F}\right)=2$.

Consider now the remaining case $h^{0}\left(L_{f}\right)=5=g$. Since $L \cdot f=2 g-2=8$, it thus follows (see, e.g., $[6,(1.4 .6)])$ that $L_{f} \approx K_{f}$. Therefore $K_{F \mid f} \approx \mathcal{O}_{f}$. Note that we can assume $q(F) \neq g(F)$, since otherwise $F$ would be ruled (see, e.g., [6, $\S 8.7]$ ), contradicting the additivity of the Kodaira dimension. Hence [14, (0.9)] applies to give $K_{F} \approx \mathcal{O}_{F}$. Since $h^{0}\left(L_{F}\right)=6$, we conclude that $F$ lies in $\mathbb{P}^{5}$, and Lemma (1.6) yields $12 \chi\left(\mathcal{O}_{F}\right) \geq 16$, or $\chi\left(\mathcal{O}_{F}\right) \geq 2$. Since $K_{F} \approx \mathcal{O}_{F}$ we also have $p_{g}(F)=1$ and therefore $q(F)=0$. This means that $F$ is a $K 3$ surface. Since $L \cdot f=8$, one example is the intersection of three quadrics in $\mathbb{P}^{5}$, and a standard argument (see, e.g., [13]) shows that this is it. Therefore this case, together with the case of degree $L \cdot f=7$ discussed above, leads to 2$)$, iii), iv) respectively.

It remains to show that $C:=r(X)$ is a curve of genus $g(C) \leq 1$. Let $\omega_{X / C}:=$ $K_{X} \otimes r^{*} K_{C}^{-1}$ be the relative dualizing sheaf of $r: X \rightarrow C$. Note that the direct image sheaf $r_{*} \omega_{X / C}$ is an invertible sheaf. Indeed, since the general fiber of $r$ is a $K 3$ surface, we have that $r_{*} \omega_{X / C}$ is a rank one torsion free sheaf. Since $C$ is smooth, $r_{*} \omega_{X / C}$ is locally free.

From a theorem of Fujita $[20,(2.41)]$ it follows that $r_{*} \omega_{X / C}$ is nef on $C$. Thus if $g(C) \geq 2$ we have that $h^{0}\left(K_{C} \otimes r_{*} \omega_{X / C}\right) \geq g(C)-1 \geq 1$. Note that

$$
h^{0}\left(K_{C} \otimes r_{*} \omega_{X / C}\right)=h^{0}\left(r_{*} K_{X}\right)=h^{0}\left(K_{X}\right) .
$$

This contradicts our working assumption that $h^{0}\left(K_{X}\right)=0$.

Q.E.D.

\section{Degree Bounds}

We follow the notation in $\S 2$. Let

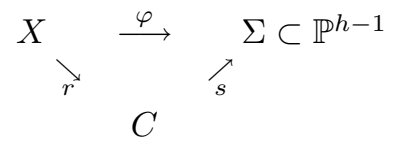


be the Remmert-Stein factorization of the rational map $\varphi: X \rightarrow \Sigma$ associated to $\left|K_{X}+L\right|, \Sigma$ an irreducible curve, as in (2.1), where $h:=h^{0}\left(K_{X}+L\right)$. Note that $s$ can also be regarded as the finite-to-one part of the Remmert-Stein factorization of the rational map associated to $K_{\widehat{X}}+\widehat{L}$. Let $F$ be a general fiber of $\varphi$ and let $f=F \cap S$ be the transversal intersection of $F$ with a smooth surface $S \in|L|$. Let $g:=g(f)$.

Let $t:=\# s$ be the sheet number of the morphism $s$. Note that since there are no nondegenerate curves in $\mathbb{P}^{h-1}$ of degree less than $h-1$, we have $\operatorname{deg} s^{*} \mathcal{O}_{\mathbb{P}^{h-1}}(1)=\lambda$ for some integer $\lambda \geq t(h-1)$. Therefore

$$
\mathcal{K}:=K_{X}+L \approx \lambda F+\mathcal{E}, \quad \lambda \geq t(h-1) .
$$

Exactly the same argument used to prove relations (5.1), ii), iii), iv) gives

$$
\begin{aligned}
d_{3} & =\mathcal{K}^{3} \geq t(h-1)(g-2) \\
d_{2} & =\mathcal{K}^{2} \cdot L \geq t(h-1)(2 g-2) ; \\
d_{1} & =\mathcal{K} \cdot L^{2} \geq t(h-1) L^{2} \cdot F+2 .
\end{aligned}
$$

We need the following three lemmas.

Lemma 7.1. If $s$ is not an embedding then $g(C) \geq t(h-1) / 2$.

Proof. Assume $\lambda>2 g(C)$. Then the degree $\lambda$ line bundle $s^{*} \mathcal{O}_{\mathbb{P}^{h-1}}(1)$ on $C$ is very ample. Since $K_{X}+L \approx \lambda F+\mathcal{E}$, this implies that the morphism $s: C \rightarrow \mathbb{P}^{h-1}$ is an embedding. Thus we conclude that if $s$ is not an embedding, then $2 g(C) \geq \lambda \geq$ $t(h-1)$. Therefore $g(C) \geq \frac{t(h-1)}{2}$.

Q.E.D.

Lemma 7.2. Assume that $S$ is a smooth ample divisor on a three dimensional connected projective manifold $X$. Assume that $S$ is a surface of general type and that there exists a morphism $r: S \rightarrow C$ with connected fibers onto a smooth curve $C$ of genus $g(C)$. Then the topological Euler characteristic of $S, e(S)$, satisfies the condition $e(S) \geq 4(g(C)-1)(g(f)-1)+1$, where the curve $f$ of genus $g(f)$ is a general fiber of $r$.

Proof. Since $S$ is of general type, $g(f) \geq 2$. We can assume that $g(C) \geq 1$, since otherwise the result is trivial. The inequality $e(S) \geq 4(g(C)-1)(g(f)-1)$ is classical (see, e.g., $[1,(11.6)])$. If we have equality, we conclude from $[1,(11.5)]$ and $g(f) \geq 2$ that $S$ is a $K(\Pi, 1)$, which is impossible by a result of the second author [6, Corollary 5.1.6].

Q.E.D.

Lemma 7.3. Let $r: X \rightarrow C$ be a morphism with connected fibers from a projective threefold $X$ onto a smooth curve $C$. If $h^{1}\left(\mathcal{O}_{F}\right)=0$ for a general fiber $F$ of $r$, then $h^{2}\left(\mathcal{O}_{X}\right) \leq h^{2}\left(\mathcal{O}_{F}\right)$.

Proof. We must show that an element of $H^{2}\left(\mathcal{O}_{X}\right)$ that goes to zero in $H^{2}\left(\mathcal{O}_{F}\right)$ is zero. By duality, it suffices to show that a holomorphic 2 -form $\omega$ on $X$ that goes to zero in $H^{0}\left(K_{F}\right)$ is zero.

Let $T_{F}, T_{X}$ and $N_{F}$ be the tangent bundles of $F, X$ and the normal bundle of $F$ in $X$ respectively. Note that since $\omega$ goes to zero in $H^{0}\left(K_{F}\right)$, the image of $\omega$ in $H^{0}\left(\wedge^{2} T_{X \mid F}^{*}\right)$ lies in $H^{0}\left(T_{F}^{*} \otimes N_{F}^{*}\right)$. Since $T_{F}^{*} \otimes N_{F}^{*} \cong T_{F}^{*}$ and since by assumption $h^{0}\left(T_{F}^{*}\right)=0$, the image of $\omega$ in $H^{0}\left(\wedge^{2} T_{X \mid F}^{*}\right)$ is zero.

Since $\omega$ is closed and represents an element of $H^{2}(X, \mathbb{C})$, the image of $\omega$ in $H^{0}\left(K_{F^{\prime}}\right)$ for any other smooth fiber $F^{\prime}$ of $r$ is also zero. By the argument of the 
last paragraph we conclude that the image of $\omega$ in $H^{0}\left(\wedge^{2} T_{X \mid F^{\prime}}^{*}\right)$ is zero for the dense set of smooth $F^{\prime}$. Thus $\omega$ is identically zero.

Q.E.D.

Theorem 7.4. Let $(\widehat{X}, \widehat{L})$ be a smooth threefold of log-general type polarized by a very ample line bundle $\widehat{L}$. Let $(X, L)$ be the first reduction of $(\widehat{X}, \widehat{L})$. Let $\varphi=s \circ r$ be the Remmert-Stein factorization of $\varphi$, the rational map associated to $K_{X}+L$. Assume that the image of $r$ is a curve, $C$. Then $s$ is an embedding unless $g(C)>0$. If $g(C)>0$, then $\varphi$ and $r$ are morphisms and the sheet number of $s$ is $t \leq 2$. If $t=2$, then $\chi\left(\mathcal{O}_{S}\right) \leq 2, h^{0}\left(K_{X}+L\right)=2$, and $3 \leq g(f) \leq 4$, where $f=F \cap S$ is the transversal intersection of a general fiber $F$ of $r$ with a general member $S \in|L|$ as in (8).

Proof. With the notation as above, if $\varphi: X \rightarrow \Sigma$ is not a morphism then by Lemma (2.2) the curve $C$ in (25) is $\mathbb{P}^{1}$. In this case $s$ is an embedding. Thus we can assume without loss of generality that $g(C)>0$ and that $\varphi$ is a morphism. As in the proof of (5.1), iv), we can assume that the restriction $L_{F}$ is very ample and $K_{F}+L_{F}$ is nef and big, without loss of generality. Thus the well known classification of polarized surfaces of sectional genus $\leq 2$ (see, e.g., $[6, \S 10.2]$ ) shows that we can assume

$$
g(f) \geq 3 .
$$

Let $h:=h^{0}\left(K_{X}+L\right)$, let $\delta:=L \cdot f$, and let $g:=g(f)$. We divide the proof in three steps.

Step I: In Step I we reduce to the case $g(f)=3$.

If $g(f) \neq 3$, then by $(27)$ we have that $g(f) \geq 4$. We claim that either

$$
\delta \geq 6 \quad \text { or } \quad \delta=5 \text { and } g=6 .
$$

Indeed, if $f \subset \mathbb{P}^{2}$ and $\delta \leq 5$ then Castelnuovo's bound (1.9) yields $g \leq \operatorname{Castel}(5,2)=$ 6 . Then, by the genus formula, either $g=6$ and $\delta=5$, or we find a contradiction. Thus we can assume $f \subset \mathbb{P}^{N}, N \geq 3$. If $\delta \leq 5$, Castelnuovo's bound gives the contradiction $g \leq \operatorname{Castel}(5,3)=2$.

Assume $t \geq 3$ and $L \cdot f \geq 6$. Then, from (26),

$$
\begin{aligned}
& d_{3} \geq 3(h-1)(g-2) \geq 6(h-1) ; \\
& d_{2} \geq 3(h-1)(2 g-2) \geq 18(h-1) ; \\
& d_{1} \geq 3 \delta(h-1)+2 \geq 18(h-1)+2 .
\end{aligned}
$$

From this, Lemma (7.1), and Lemma (7.2) we have

$$
\chi\left(\mathcal{O}_{S}\right)=\frac{d_{2}+e(S)}{12} \geq\left\lceil\frac{18(h-1)+6(3 h-5)+1}{12}\right\rceil=3 h-3 .
$$

Thus Tsuji's inequality with the inequality $(29)$ gives

$$
h \geq \frac{3(h-1)}{4}+\frac{1}{12}+\frac{3(h-1)}{32}+\frac{3 h-3}{2},
$$

or $h<2$. This contradicts $h \geq 2$.

Now we do the case when $t \geq 3, g=6$, and $\delta=5$. In this case $d_{1} \geq 15(h-1)+2$, $d_{3} \geq 12(h-1)$, and $d_{2} \geq 30(h-1)$ by $(26)$. As above, Tsuji's inequality with Lemma (7.1) and Lemma (7.2) gives

which contradicts $h \geq 2$.

$$
h \geq \frac{5(h-1)}{8}+\frac{1}{12}+\frac{3(h-1)}{16}+\frac{5 h-6}{2},
$$


Thus we can now assume $t=2$ and $\delta=L \cdot f \geq 6$. Then, from (26),

$$
\begin{aligned}
& d_{3} \geq 2(h-1)(g-2) \geq 4(h-1) \\
& d_{2} \geq 2(h-1)(2 g-2) \geq 12(h-1) \\
& d_{1} \geq 2 \delta(h-1)+2 \geq 12(h-1)+2 .
\end{aligned}
$$

From this, Lemma (7.1), and Lemma (7.2) we have

$$
\chi\left(\mathcal{O}_{S}\right)=\frac{d_{2}+e(S)}{12} \geq\left\lceil\frac{12(h-1)+12(h-2)+1}{12}\right\rceil=2 h-2 .
$$

Thus Tsuji's inequality with the inequality (31) gives

$$
h \geq \frac{h-1}{2}+\frac{1}{12}+\frac{h-1}{16}+h-1,
$$

or $h \leq 2$. Since $h \geq 2$ we have $h=2$. Checking Tsuji's inequality, we see that $\chi\left(\mathcal{O}_{S}\right) \leq \frac{21 h+23}{24}<3$ in this case. Thus if $g=g(f) \geq 6$, and recalling (30), we have the contradiction to Miyaoka's inequality

$$
18=9 \chi\left(\mathcal{O}_{S}\right) \geq d_{2} \geq 4(g(f)-1) .
$$

To see that $g(f)=5$ is not possible, note that in this case we have $\delta \geq 7$ by Castelnuovo's bound for the genus. Thus since $h=2$ we have, from (30), $d_{3} \geq 6$, $d_{2} \geq 16$ and $d_{1} \geq 16$. Using this, $h=2$, and $\chi\left(\mathcal{O}_{S}\right) \leq 2$, we conclude from Proposition (1.5), with $\widehat{d}:=\widehat{L}^{3}$, that (note that in (1.5) we can assume $h^{0}\left(K_{\widehat{X}}\right)=0$ since otherwise $\left|K_{X}+L\right|$ would give a birational map)

$$
\widehat{d}(\widehat{d}-20) \geq-208+12 d_{2}+17 d_{1}+d_{3} \geq 262 .
$$

This gives $\widehat{d} \geq 30$ and hence $d:=L^{3} \geq \widehat{d} \geq 30$. By the Hodge index theorem, $480 \leq d d_{2} \leq d_{1}^{2}$, which implies that $d_{1} \geq 22$. But using this, and $\chi\left(\mathcal{O}_{S}\right) \geq 2$ from (31), Tsuji's inequality gives a contradiction:

$$
2=h \geq \frac{6}{64}+\frac{22}{24}+\frac{\chi\left(\mathcal{O}_{S}\right)}{2} \geq \frac{193}{92}>2 .
$$

We are left with the case when $t=2, g=6$, and $\delta=5$. In this case $d_{1} \geq 10(h-1)+2$, $d_{3} \geq 8(h-1)$ and $d_{2} \geq 20(h-1)$ by $(26)$. As above, Tsuji's inequality with Lemma (7.1) and Lemma (7.2) gives

$$
h \geq \frac{5(h-1)+1}{12}+\frac{h-1}{8}+\frac{20(h-1)+20(h-2)+1}{24} .
$$

This implies $h \leq 2$, and hence that $h=2$. Checking Tsuji's inequality, we see that $\chi\left(\mathcal{O}_{S}\right) \leq 2$ in this case. This again contradicts Miyaoka's inequality $9 \chi\left(\mathcal{O}_{S}\right) \geq$ $d_{2} \geq 20(h-1)=20$.

Step II: Assume now that $\kappa(X) \geq 0$. Thus from [5, (3.3)] we have that $h \geq 6$ (cf. (4.5)). By Step I we can assume that $g=3$.

Since $K_{F}+L_{F}$ is nef and big, we see from [6, Theorem 10.2.7] that $F$ is birational to a $K 3$ surface. Then $h^{1}\left(\mathcal{O}_{F}\right)=0, h^{2}\left(\mathcal{O}_{F}\right)=1$, so that Lemma (7.3) yields $h^{2}\left(\mathcal{O}_{S}\right) \leq h+1$. Therefore we have by Lemma (7.1)

$$
\chi\left(\mathcal{O}_{S}\right) \leq h+1-h^{1}\left(\mathcal{O}_{S}\right)+1 \leq h+1-g(C)+1 \leq h+2-\frac{t(h-1)}{2} .
$$


Thus if $t \geq 2$, then $\chi\left(\mathcal{O}_{S}\right) \leq 2$. Since $g \geq 3$, by using Lemma (7.1) and Lemma (7.2) we also have for $t \geq 2$, as in Step I,

$$
\begin{aligned}
\chi\left(\mathcal{O}_{S}\right) & =\frac{d_{2}+e(S)}{12} \\
& \geq \frac{t(h-1)(2 g-2)+(t(h-1)-2)(2 g-2)}{12} \\
& \geq \frac{4 h-6}{5} .
\end{aligned}
$$

This implies the contradiction that $h \leq 3$. Therefore we conclude that $t=1$.

Step III: We are now left with the case when $g=g(f)=3$ and $\kappa(X)=-\infty$.

From the exact sequence $0 \rightarrow K_{X} \rightarrow K_{X} \otimes L \rightarrow K_{S} \rightarrow 0$ and $h^{0}\left(K_{X}\right)=0$ we have $h \leq h^{2}\left(\mathcal{O}_{S}\right)$. Since $g(C) \geq 1$ we have by the additivity of Kodaira dimension that $h^{2}\left(\mathcal{O}_{F}\right)=0$. From [6, Theorem 10.2.7] and the bigness of $K_{F}+L_{F}$ for a general $F$ we see that $h^{1}\left(\mathcal{O}_{F}\right)=0$. Then Lemma $(7.3)$ gives $h^{1}\left(K_{X}\right)=0$, and hence $h \geq h^{2}\left(\mathcal{O}_{S}\right)$. Therefore we conclude that $h=h^{2}\left(\mathcal{O}_{S}\right)$, and hence $\chi\left(\mathcal{O}_{S}\right)=$ $h-h^{1}\left(\mathcal{O}_{S}\right)+1$. Thus we have, by Lemma (7.1),

$$
\chi\left(\mathcal{O}_{S}\right)=h-h^{1}\left(\mathcal{O}_{S}\right)+1 \leq h-g(C)+1 \leq h+1-\frac{t(h-1)}{2} .
$$

Assume $t \geq 2$. Then $\chi\left(\mathcal{O}_{S}\right) \leq 2$. Since $K_{S}^{2}=d_{2} \geq t(h-1)(2 g-2)$, by using Lemma (7.1) we also have

$$
\chi\left(\mathcal{O}_{S}\right)=\frac{K_{S}^{2}+e(S)}{12} \geq\left\lceil\frac{t(h-1)(2 g-2)+(t(h-1)-2)(2 g-2)+1}{12}\right\rceil .
$$

Since $g=3$ and $t \geq 2$, we conclude that $\chi\left(\mathcal{O}_{S}\right) \geq \frac{4 h-5}{3}$. Since $\chi\left(\mathcal{O}_{S}\right) \leq 2$, it follows that $h \leq 2$ and hence $h=2$.

If $t \geq 3$ then, by Lemma (7.1), we have that $g(C) \geq 2$. Thus $\chi\left(\mathcal{O}_{S}\right) \leq 1$. Since $S$ is of general type we conclude that $\chi\left(\mathcal{O}_{S}\right)=1$. But $d_{2} \geq 12$ by formulas (26). This contradicts Miyaoka's inequality. Thus $t \leq 2$.

Remark 7.5. Notation as in (7.4). One immediate consequence of the proof of (7.4) is that the sheet number of $s$ is $t=1$ if $\kappa(X) \geq 0$.

\section{The CASE OF $\operatorname{dim} \widehat{X} \geq 4$}

Let $\widehat{L}$ be a very ample line bundle on a smooth, $n$-dimensional, projective manifold $\widehat{X}$ with $n \geq 4$. Assume that the Kodaira dimension of $K_{\widehat{X}}+(n-2) \widehat{L}$ is at least 3 . Then by the main result of [5], $h^{0}\left(K_{\widehat{X}}+(n-2) \widehat{L}\right) \geq 2$. Let $\varphi: \widehat{X} \rightarrow \Sigma$ denote the meromorphic map associated to $\left|K_{\widehat{X}}+(n-2) \widehat{L}\right|$, and assume that the image of $\widehat{X}$ under $\varphi$ is 1-dimensional.

Let $Y$ be the transversal intersection of $n-3$ general elements of $|\widehat{L}|$. It is a classical result of the second author [6, Theorem 5.2.1] that any morphism of $Y$ to a curve extends to a morphism from $\widehat{X}$ to the same curve. From this we get immediate extensions of our results to the case of $n \geq 4$ dimensions.

Theorem 8.1. Let $\widehat{L}$ be a very ample line bundle on a smooth, $n$-dimensional, projective manifold $\widehat{X}$ with $n \geq 3$. Assume the Kodaira dimension of $K_{\widehat{X}}+(n-2) \widehat{L}$ is at least 3. Let $r: \widehat{X} \rightarrow C$ be the morphism with connected fibers (see (2.1)) from the Remmert-Stein factorization of $\varphi=s \circ r$, the meromorphic map associated to 
$\left|K_{\widehat{X}}+(n-2) \widehat{L}\right|$. If either $h^{0}\left(K_{\widehat{X}}+(n-2) \widehat{L}\right) \geq 7$, or $\kappa\left(K_{\widehat{X}}+(n-3) \widehat{L}\right) \geq 0$, or $g(C) \geq 1$, then $\varphi$ is a morphism.

Since the map $\Gamma\left(K_{\widehat{X}}+(n-2) \widehat{L}\right) \rightarrow \Gamma\left(K_{Y}+\widehat{L}_{Y}\right)$ is onto, the results of the last section extend immediately to the case of $n \geq 4$. One slight improvement can be made in Theorem (7.4) in this case. The case $t=2, g(f)=4$ can be shown not to exist. The key point is that the classification [6, Theorem 11.6.1] of projective manifolds with genus 4 hyperplane sections can be used to show that the general fibers of the restriction $r_{Y}: Y \rightarrow C$ in this case cannot be hyperplane sections of any threefolds.

Note added in proof. The argument used to show inequalities i) in (3.1) is incorrect. Indeed from Proposition (2.4) we can only conclude $L \cdot f \geq \sqrt{d(f \cdot f)} \geq 5$. But in fact $L \cdot f \geq \sqrt{d(f \cdot f)} \geq 6$ holds true as stated. Here there is the correct argument. First note that we are working under the assumption $h \geq 6$ (see Theorem (3.4)). The Hodge index inequality gives $\left(K_{S} \cdot f\right)^{2} \geq(f \cdot f)\left(K_{S} \cdot K_{S}\right) \geq 2 K_{S} \cdot K_{S}$. From (3.1), iv) we get $K_{S} \cdot K_{S} \geq(h-1)^{2}(f \cdot f) \geq 50$. Thus we conclude $K_{S} \cdot f \geq 10$. Then the genus formula gives $2 g(f)-2 \geq K_{S} \cdot f+f \cdot f \geq 12$, or $g(f) \geq 7$. If $L \cdot f \leq 5$ the Castelnuovo bound (1.9) would give the contradiction $g(f) \leq 6$.

\section{REFERENCES}

1. W. Barth, C. Peters, and A. Van de Ven, Compact Complex Surfaces, Ergeb. Math. Grenzgeb. (3) 4 (1984), Springer, Berlin. MR 86c:32026

2. A. Beauville, "L'application canonique pour les surfaces de type général," Invent. Math. 55 (1979), 121-140. MR 81m:14025

3. M.C. Beltrametti, M. Schneider, and A.J. Sommese, "Threefolds of degree 11 in $\mathbb{P}^{5}$," in Complex Projective Geometry, ed. by G. Ellingsrud, C. Peskine, G. Sacchiero, and S.A. Stromme, London Math. Soc. Lect. Notes, 179 (1992), 59-80. MR 94d:14037

4. M.C. Beltrametti, M. Schneider and A.J. Sommese, Some special properties of the adjunction theory for threefolds in $\mathbb{P}^{5}$, Memoirs of the Amer. Math. Soc., vol. 116, n. 554 (1995). MR 95k:14055

5. M.C. Beltrametti and A.J. Sommese, "On the dimension of the adjoint linear system for threefolds," Ann. Scuola Norm. Sup. Pisa Cl. Sci. Ser. (4), XXII (1995), 1-24. MR 96e: 14005

6. M.C. Beltrametti and A.J. Sommese, The Adjunction Theory of Complex Projective Varieties, Expositions in Mathematics 16 (1995), W. de Gruyter, Berlin. MR 96f:14004

7. T. Fujita, "Theorems of Bertini type for certain types of polarized manifolds," J. Math. Soc. Japan 34 (1982), 709-718. MR 84d:14003

8. J. Harris and D. Eisenbud, Curves in Projective Spaces, S.M.S. Université de Montreal, (1982). MR 84g:14024

9. R. Hartshorne, Algebraic Geometry, Graduate Texts in Math. 52 (1977), Springer, New York. MR 57:3116

10. P. Ionescu, "Embedded projective varieties of small invariants," in Proceedings of the 1982 Week of Algebraic Geometry, Bucharest, ed. by L. Badescu and D. Popescu, Lecture Notes in Math. 1056 (1984), 142-187, Springer, New York. MR 85m:14024

11. Y. Kawamata, K. Matsuda, and K. Matsuki, "Introduction to the minimal model problem," in Algebraic Geometry, Sendai 1985, ed. by T. Oda, Advanced Studies in Pure Mathematics 10 (1987), 283-360. MR 89e:14015

12. A. Lanteri, M. Palleschi, and A.J. Sommese, "On triple covers of $\mathbb{P}^{n}$ as very ample divisors," in Classification of Algebraic Varieties, Proceedings L'Aquila, 1992, ed. by C. Ciliberto, E.L. Livorni, and A.J. Sommese, Contemp. Math. 162 (1994), 277-292. MR 95f:14012

13. E.L. Livorni, "Classification of algebraic nonruled surfaces with sectional genus less than or equal to six," Nagoya Math. J. 100 (1985), 1-9. MR 87c:14043

14. A.J. Sommese, "Hyperplane sections of projective surfaces, I: The adjunction mapping," Duke Math. J. 46 (1979), 377-401. MR 82f:14033 
15. A.J. Sommese, "Hyperplane Sections," in Algebraic Geometry, Chicago Proceedings, 1980, ed. by A. Libgober and P. Wagreich, Lecture Notes in Math. 862 (1981), 232-271, Springer, New York. MR 83i:14009

16. A.J. Sommese, "On the minimality of hyperplane sections of projective threefolds," J. Reine Angew. Math. 329 (1981), 16-41. MR 83j:14038

17. A.J. Sommese, "On the adjunction theoretic structure of projective varieties," in Complex Analysis and Algebraic Geometry, Proceedings Göttingen 1985, ed. by H. Grauert, Lecture Notes in Math. 1194 (1986), 175-213, Springer, New York. MR 87m:14049

18. A.J. Sommese, "On the nonemptiness of the adjoint linear system of a hyperplane section of a threefold," J. Reine Angew. Math. 402 (1989), 211-220; "Erratum," J. Reine Angew. Math. 411 (1990), 122-123. MR 90j:14011

19. H. Tsuji, "Stability of tangent bundles of minimal algebraic varieties," Topology 27 (1988), 429-442. MR 90d:14021

20. E. Viehweg, Quasi-Projective Moduli for Polarized Manifolds, Ergeb. Math. Grenzgeb. (3) 30 (1995), Springer, Berlin. CMP 96:6

Dipartimento di Matematica, Università degli Studi di Genova, Via Dodecaneso 35, I-16146 Genova, ItALy

E-mail address: beltrame@dima.unige.it

Department of Mathematics, University of Notre Dame, Notre Dame, Indiana 46556

E-mail address: sommese.1@nd.edu 